\title{
Stefan Abel
}

\section{>Paulus und Thekla Ilk oder >Die guldin regek - (Pseudo-)Biographie eines Beichtigers}

\begin{abstract}
This article deals with a dialogue tract, surviving in one manuscript from 1469, that teaches a female penitent seven golden rules of exemplary piety; a contemporary short version of them is published here. The catechetic programme of the tract is embedded in the biography of a prototypical Dominican father confessor and makes use of the apocryphal legend of Saint Paul and Thecla as a biographical model. The anonymous author also bases his text on a certain type of mystical autobiography and, possibly, the Legend of Meister Eckhart's Daughter. Much as the text appears to allude to real figures of the Dominican Order, its historical truth cannot be confirmed.
\end{abstract}

DOI 10.1515/bgsl-2014-0050

\section{I. sPaulus und Theklaı I oder II?}

Unter dem gemeinsamen, nicht mittelalterlichen Titel >Paulus und Thekla ${ }^{1}$ sind zwei geistliche, bislang unedierte Texte des 15. Jahrhunderts geläufig, die auf irrtümliche Weise in nächste, sogar namentliche Nähe zueinander gerückt sind. Der alte Bibliothekskatalog (1455-1457) des Dominikanerinnenklosters St. Katharina in Nürnberg enthält unter der Signatur E XXXVII (Sachgruppe der Predigten) einen Eintrag, den Spamer ${ }^{2}$ und mit ihm Stammler ${ }^{3}$ auch auf $>$ Paulus und Thekla II bezogen. Die Buchmeisterin und Schreiberin Kunigunde Niklasin († 1457), die >Paulus und Thekla II « vermutlich gar nicht kannte, verzeichnete auf

1 Siehe Kurt Ruh: [Art.] Paulus und Thekla I und II, in: ${ }^{2}$ VL, Bd. 7, 1989, Sp. 390-393 sowie Sabina Foidl: [Art.] Paulus und Thekla I und II, in: Wolfgang Achnitz (Hg.): Deutsches Literatur-Lexikon. Das Mittelalter, Bd. 2: Das geistliche Schrifttum des Spätmittelalters. Mit einem einführenden Essay von Regina D. Schiewer und Werner Williams-Krapp, Berlin u. Boston 2011, Sp. $1084 \mathrm{f}$.

2 Vgl. Adolf Spamer: Zur Überlieferung der Pfeiffer'schen Eckeharttexte, in: PBB 34 (1909), S. 307-420, hier S. 378.

3 Vgl. Wolfgang Stammler (Hg.): Deutsche Philologie im Aufriss, Bd. 2, Berlin 1960, Sp. 977.

Dr. Stefan Abel: Universität Bern, Institut für Germanistik, Länggassstr. 49, CH-3000 Bern 9, E-Mail: stefan.abel@germ.unibe.ch 
Bl. $105^{\mathrm{v}}$ des von ihr zusammengestellten Katalogs (Nürnberg, Stadtbibliothek, Cod. Cent. VII, 79) jedoch ausschließlich >Paulus und Thekla I<:

[...] Item ein news puch; das helt in im | von IIII zukunft unsers herrn und III | predig, und wie unser herr ein furman und ein | kaufman was, und wie ein peichtvater | seiner gaistlichen peichttochter gelobet, | das er wolt Paulus sein und sy Thekla in | gotlicher lieb, und von dem heiligen sacrament | und von den selen und fegfewr und von dem | helfen der selen und predig und gepet und | frog und von gedancken und vil guter | matery etc. | Das puch hat swester Kungunt Niklasin | geschriben. ${ }^{4}$

Die so katalogisierte Handschrift - Nürnberg, Stadtbibliothek, Cod. Cent. VI, $431^{5}$ von 1446, die in genau dieser textlichen Zusammenstellung auf uns gekommen ist, überliefert >Paulus und Thekla I unikal $\left(\mathrm{Bl} .51^{\mathrm{r}}-75^{\mathrm{v}}\right)$. Es handelt sich dabei um einen schlichten, aszetischen Dialogtraktat, der auf traditioneller Väterlehre beruht und, ausgehend von Gal 5,17, die darin enthaltene Lehre vor allem allegorisch vermittelt. Als ein news puch gelangte die Handschrift nicht etwa als Geschenk oder Mitgift von außen ins Katharinenkloster, sondern wurde dort von der Buchmeisterin selbst in Nürnbergischer Schreibsprache geschrieben. Eine Verwechslung beider Texte war leichthin möglich, da sie - >Paulus und Thekla I durchwegs, >Paulus und Thekla IK allerdings nur einmalig - auf die titelgebende Beziehung zwischen dem Apostel Paulus und der von ihm bekehrten Thekla von Ikonium Bezug nehmen, geschildert in den apokryphen Paulusakten. Zudem gehen sie gleichermaßen von einem biblischen Motto aus - Mt 19,17 in >Paulus und Thekla II - und sind dialogisch strukturiert: Beichtvater und Beichttochter im Gespräch. Um einer weiteren Verwechslung auch in diesem Beitrag entgegenzuwirken, ersetze ich >Paulus und Thekla II , der als bislang wenig beachteter Text hier im Mittelpunkt steht, durch den handschriftlich verbrieften Titel `Die guldin regel (GR), unikal überliefert in Frankfurt/Main, Universitätsbibliothek, Ms. Praed. 159, Bl. $77^{\mathrm{r}}$ (um 1469) ${ }^{6}$.

4 Bayerische Akademie der Wissenschaften in München (Hg.): Mittelalterliche Bibliothekskataloge Deutschlands und der Schweiz [MBK], Dritter Band/Dritter Teil: Bistum Bamberg, bearb. v. Paul Ruf, München (2. ND 1969), S. 608, Z. 12-18.

5 Ausführliche Beschreibung bei Karin Schneider: Die deutschen mittelalterlichen Handschriften. Beschreibung des Buchschmucks: Heinz Zirnbauer, Wiesbaden 1965 (Die Handschriften der Stadtbibliothek Nürnberg I), S. 107-111.

6 Die Handschrift liegt digitalisiert vor (http://sammlungen.ub.uni-frankfurt.de/msma/content/ titleinfo/4480585, Aufrufdatum: Oktober 2014). Die im Folgenden transkribierten Textstellen aus Ms. Praed. 159 und anderen Handschriften sind modern interpunktiert und hinsichtlich Groß- und Kleinschreibung geregelt (Personen- und Ortsnamen sowie Wörter am Satzanfang groß). Mit Ausnahme etwa des Schaft-s, das zu rundem $s$ vereinheitlicht ist, sind die Textstellen möglichst handschriftengetreu wiedergegeben. Auslassungen und offensichtliche Fehler sind recte gesetzt. 


\section{II. 'Die guldin regel - Katechese entlang der Biographie}

Dieser mittelalterliche Werkstitel ist für sich allein schon Ausdruck der besonderen Gültigkeit eines jeden, mit ihm betitelten Werkes. Er bezieht sich sowohl in der GR, als auch in den später vorgestellten Textzeugen einer entsprechenden Kurzfassung jedoch ausdrücklich nicht auf diejenige Goldene Regel, die biblisch in Mt 7,21 (Summe des Gesetzes) und Lc 6,31 (Feindesliebe) zugrunde gelegt und seit dem 18. Jahrhundert in der Formulierung >Was du nicht willst, dass man dir tu', das füg' auch keinem andern zu populär geworden ist. Ein dennoch mögliches Bindeglied zwischen der biblischen Goldenen Regel und dem vorliegenden Dialogtraktat ist die christliche Apologetik, für die diese Regel ein Beweismittel für die Vernünftigkeit christlicher Lehre darstellt, da letztere mit natürlicher Sittlichkeit harmoniere; ${ }^{7}$ das `Decretum Gratiani` (um 1140) betrachtet die Goldene Regel als Ausdruck des in lege et Evangelio enthaltenen Naturrechts. Die GR stellt folglich, allerdings nur vereinzelt, den Vorzug des Christentums gegenüber dem Judentum heraus und kündigt, zumindest eingangs, die Abhandlung von sieben `Geboten`, `Gesetzen ` oder auch >Rechten` an, darunter des Naturrechts an erster Stelle.

Tatsächlich bespricht die GR in ihren sieben Kapiteln, die in Ms. Praed. 159 oftmals sauber auf einer Seite abschließen, sieben Arten von gebotten, die es vom gläubigen Christen einzuhalten gilt. Sie zieht dafür, nebst nur wenig namentlich genannten Autoritäten ${ }^{8}$, in der Hauptsache die Heilige Schrift autorisierend heran:

Hie ist aber zewissent, das der gebotten sind siben. Das erst ist ain gebott der natur. Das ander ist ain gebott der geschribnen gesaczt gottes der alten ee vnd der nüwen. - Wenn was die alten halten nach dem buchstaben, das halten wir nach dem gaistlichen sinn. - Das dritt gebott der obersten, die vnser ler vnd tugent ordenlich [Prädikat fehlt]. Das fierd gebott ist von vswendiger gelüt vnd eyd. Das funft gebott ist des jnwendigen menschen der gewissne, die ümer bindet.

7 »Die positiv gefaßte Goldene Regel schließt in Mt 7,12 den Hauptteil der Bergpredigt $(5,17-7,12)$ zusammenfassend ab. In der Wendung >Gesetz und Propheten $>(7,12 b)$ wird Mt 5,17-20 wiederaufgenommen und damit die Goldene Regel in den Zusammenhang der Frage nach der >besseren` Gerechtigkeit der Christen gestellt« (Gerhard Müller [u. a.] [Hgg.]: Theologische Realenzyklopädie, Bd. 13, 1984, S. 573).

8 Es sind dies Aristoteles (`De animaく, Bl. $77^{\mathrm{r}}$ ), das Dekret `Omnis utriusque sexus` des Vierten Laterankonzils von 1215, das die jährliche Beichte verordnet (Bl. $88^{\mathrm{r}}$; hier in einem Zug mit der Bulle `Extra de sepulturis` [1299] von Papst Bonifatius VIII. genannt), Hugo von St. Viktor (`De fructibus carnis et spiritus`, Bl. 96 ${ }^{\mathrm{r}}$ ), Bernhard von Clairvaux (Bl. $97^{\mathrm{V}}$ und Bl. 102 $\mathrm{r}$ ), Altväter (Bl. $124^{\mathrm{v}}-125^{\mathrm{r}}$ ), Seneca (Bl. $121^{\mathrm{r}}$, Bl. $141^{\mathrm{v}}$ und Bl. 143) und Pseudo-Anselm von Canterbury (`Admonitio morienti<, Bl. $145^{\mathrm{v}}-146^{\mathrm{r}}$ ). 
Wenn das sechst gebott ist der rechtbücher vnd der vffsacz der hailigen kirchen. Das sibend gebott ist gůte alte gewonhait, die ain recht ist worden $\left(\mathrm{Bl} .77^{\mathrm{v}}-78^{\mathrm{r}}\right)$.

Diese Gebote werden jedoch nicht konsequent besprochen. Vielmehr ist ihre Abhandlung von der Vermittlung vorbildlicher Verhaltensweisen der Frömmigkeitspraxis überlagert, welche die eigentlichen sieben Goldenen Regeln ausmachen und die sich so auch in der Kurzfassung des Motivs der sguldin regel niedergeschlagen haben (siehe ab S. 649). So fehlen explizite Ausführungen etwa zum Naturrecht oder dem Gelübde gänzlich; Gebote der Obrigkeit werden kurz aufgegriffen, als sich die Tochter beim Beichtvater danach erkundigt, wie sol ich die tugent üben nach dem gebot mins obrosten $\left(\mathrm{Bl} .116^{\mathrm{r}-\mathrm{v}}\right)$. Es ergibt sich daraus folgende Gegenüberstellung von Geboten und Frömmigkeitspraxis:

\section{Gebote}

1. Naturrecht

2. Gebote des Alten und Neuen Bundes

3. Gebote der Obrigkeit

4. Gelübde

5. Gewissen

6. Welt- und Kirchenrecht

7. Gewohnheitsrecht

\section{Frömmigkeitspraxis}

$$
\begin{aligned}
& \rightarrow \text { häufige Beichte } \\
& \rightarrow \text { Vermeidung von Sünde } \\
& \rightarrow \text { Tugendübungen } \\
& \rightarrow \text { häufige Kommunion } \\
& \rightarrow \text { Selbstbetrachtung } \\
& \rightarrow \text { Geduld im Leiden, Aufgabe des Eigenwillens } \\
& \rightarrow \text { Vorbereitung auf den Tod }
\end{aligned}
$$

Die Gliederung der Gebote orientiert sich zum Teil an Thomas' von Aquin ıSumma theologiae، (I-II q. 90-108) - lex naturalis (1.), divina (2.), humana (6.) -, ergänzt um dem Menschen von außen bzw. oben oktroyierte (3.), sich nach außen hin selbst auferlegte (4.) und verinnerlichte Gebote (5.) sowie um das Sterben als einem seit Anbeginn der Menschheit wirkenden `Naturgesetz` (7.). Die gewissermaßen klimaktisch strukturierte Verknüpfung der einzelnen Gebote mit Facetten der Frömmigkeitspraxis wirkt insgesamt recht unmotiviert. Nur an einer Stelle wird sie tatsächlich ausgeführt:

ıIst ietzunt besser wen dick bichten, biterlich sich vor sünden hüten sunderlichen vnd sich in tugent üben flißclich? Das erst ain gebot der naturen, das ander ist ain gebott gotes vnd der gesatzt, $d z$ drit ist ain gebot des obrosten, dem man sich in gehorsamikait gegeben haut $\left(\mathrm{Bl} .121^{\mathrm{r}-\mathrm{v}}\right)$.

Die Systematik der Gebote wird somit frömmigkeitspraktisch und heilsfördernd umgebrochen. Und begleitend zum jeweils höheren Stellenwert des einzelnen Gebotes steigern sich auch die Intensität und der Heilsnutzen des frommen Verhaltens: Dem routinierten, bald schon automatisierten Abbeichten der Sünden (Gesetzesbefolgung im Alten Bund) folgt der Ratschlag, tunlichst vorab jegliche Sünde 
$\mathrm{zu}$ vermeiden und sich nicht allein auf die reinigende Wirkung der Beichte zu verlassen (Überwindung der bloßen Gesetzesbefolgung im Neuen Bund). Analog dazu wird der gnadenvolle und häufige Vollzug der Kommunion allein (Stufe des Gelübdes) durch eine vorausgehende, intensive Kommunionsvorbereitung im Sinne der Selbstbetrachtung übertroffen (Stufe des Gewissens). Der bloßen Befolgung hetero- oder autonom gesetzter Gebote, z. B. eines Gelübdes (4. Gebot der GR), steht somit die Gewissensergründung gegenüber, das heißt, die Betrachtung eigenen Denkens, Fühlens und Verhaltens in Abgleich mit hetero- und autonom gegebener, nun aber interiorisierter Gesetzmäßigkeit. Somit begleitet die GR ihre Rezipienten auf dem Weg zu Verinnerlichung, Intensivierung und fortschreitender Verfeinerung der Frömmigkeit, die in der Abfolge der Gebote impliziert ist. Dementsprechend erläutert die GR zu Beginn den Vorzug des Christentums gegenüber dem Judentum, dem es an ganczikait der behaltnusse der gebotten ( $\mathrm{Bl} .82^{\mathrm{V}}$ ) mangle, denn die Juden behalten die gebott allain von vssnen nach der hand vnd nit von jnnen nach dem gemüt vnd nach ettlichen personen vnd nit nach allen menschen, aber wir halten si von vssnen vnd vil me von jnnen $\left(\mathrm{Bl} .83^{\mathrm{r}}\right){ }^{9}$

Die Überschrift des Dialogtraktats von der ıguldin regel nennt das auf Mt 19,17 anspielende Motto Behalt die gebott, so behaltent sy dich ouch (Bl. $77^{\mathrm{r}}$ ), das im Anschluss lateinisch zitiert sowie homilienartig und mit scholastischer Spitzfindigkeit ausgelegt wird:

$\triangle S j^{10}$ vis ad ${ }^{11}$ vitam ingredi serua mandata (Mt 19,17). Also sprach vnser lieber herr Ihesus Cristus zů ainem, der jn fraugte, wie er behalten mochte werden: ‘Wiltu ingaun in das ewig leben, so behalt die gebott. ${ }^{12}$ Es ist zů wissen, das Aristotiles sprach in >libro de anima ${ }^{13}$,vita est viuentibus esser, das ist, das leben ist den lebenden ain wesen als alle wesen irs lebens, als

9 Dies ist einer von vier antijüdischen Topoi im 1. Kapitel $\left(\mathrm{Bl} .82^{\mathrm{v}}-83^{\mathrm{V}}\right)$ : Das Christentum zeichne sich zudem durch clarhait des bekennens - wie wol wir globen, das geschehen ist, vnd sie globten, das es geschehen solte, so ist es doch ain glob. [...] Si geloupten von jnnen durch die figuren vnd in dem schatten, das wir jnnerlich gloubent vnd vsserlich verrechent $\left(\mathrm{Bl} .82^{\mathrm{v}}-83^{\mathrm{r}}\right)-$, sowie globlichhait jn der anbettung der von den Juden nicht anerkannten Trinität und luterkait in der begird $\left(\mathrm{Bl} .82^{\mathrm{v}}\right)$, da die Juden mehr dem irdisch zitlich zugeneigt seien.

10 Die Lombarde am Beginn des 1. Kapitels (Bl. $77^{\mathrm{r}}$ ) wurde nicht ausgeführt; dass ein etwas größerer Schreibraum dafür freigelassen wurde, lässt darauf schließen, dass ursprünglich wohl rubrizierte Lombarden angedacht waren.

11 Die hier in Abgleich mit dem lateinischen Bibeltext eingefügte Präposition fehlt in der Handschrift (Bl. $77^{\mathrm{r}}$ ).

12 Dieser Lehrsatz ist in das Gespräch zwischen Jesus Christus und dem reichen Jüngling eingebettet (Mt 19,16-26).

13 Aristoteles: `De animaく II,4 (Immanuel Bekker [Hg.]: Aristotelis opera, 2 Bde., Berlin 1831 ff., S. 415b). 
der stain sůchet sin wesen hie nidnen jn der schwäre vnd das für da obnen jn den lüfften. Also alles das, das leben haut, begert sins lebens, vnd nit allain des lebens, sunder och ain lang leben vnd nit allain ain lang leben, sunder och ain lang leben, gesund leben. Vnd darumb begerent wir des ewigenn lebens. Do ist ain gancz leben, ain lang, ewig leben vnd ain gesuntber, vnuermischtz leben. Nu spricht Ihesus, das ewig leben: ‘Wiltu jngaun in das leben, .... Es ist waur: Was in vns gaut, das gaut och vsser vns. Das zitlich leben gaut in vns, die sele in den lib jn dem fierden mounet. ${ }^{14}$ Die sele ist ain leben des lebens. Dasselb leben gaut och vsser vns, so wir sterbent. Darumb spricht er swilt du gaun jn das leben` vnd nit >das leben jn dich . Wilt du nun gand jn das leben, so behalt die gebott $\left(\mathrm{Bl} .77^{\mathrm{r}-\mathrm{v}}\right)$.

Dieses Motto spielt mit der reichen Polysemie des Verbs behalten: Im hiesigen Kontext hat es sowohl die Bedeutung von "(eine auferlegte oder übernommene Norm) halten, einhalten, befolgen«, als auch von »jn. erretten, erlösen; jn. der ewigen Seligkeit teilhaftig machen ${ }^{15}{ }^{15}$ Gebotebefolgung - von Seiten des gläubigen Christen - und Erlösung - von Seiten Gottes - gehen hier eine kausale Verbindung ein, die sprachlich über die Verwendung ein und desselben Verbs zum Ausdruck gebracht wird.

Beachtung verdient die GR jedoch weniger für die Darstellung von sieben Geboten und der von Beichtvater an die Beichttochter vermittelten Belehrungen über Beichte, Dekalog, Tugend, Kommunion, Leiden und Sterben. Außergewöhnlich ist vielmehr der biographische Leitfaden, der den Dialogtraktat von Beginn an durchzieht. Er verleiht ihm - demnach geradezu eine geistliche Novelle - die Rahmenhandlung, entlang der die innere Entwicklung des Beichtvaters aus der Perspektive eines impliziten Erzählers auktorial ${ }^{16}$ an entsprechend sieben Lebensstationen geschildert und vorbildliche Frömmigkeitspraxis kapitelweise vermittelt

14 Gemäß aristotelischer Sukzessivbeseelung besitzt der Fötus zunächst eine lebensspendende anima vegetativa, danach eine empfindsame anima sensitiva und schließlich erst ab dem 40 . (Männer) bzw. 90. Tag (Frauen) eine anima intellectiva, das heißt, eine vernunftbegabte Seele, die ihn letztlich zum Menschen als vernunftbegabtem Wesen macht (Aristoteles: >De historia animalium $<$ VII,3 und $>$ De generatione animalium $<$ II,3).

15 Ulrich Goebel [u. a.] (Hgg.): Frühneuhochdeutsches Wörterbuch, Bd. 3: barmherzigkeit bezwüngnis, 2002, Sp. 706 und Sp. 721.

16 Gemäß Müllers Klassifizierung von (Auto-)Biographien des Mittelalters liegt im Fall der GR eine »Er-Erzählung nach biographischem Muster« vor (Ulrich Müller: Thesen zu einer Geschichte der Autobiographie im deutschen Mittelalter, in: Margarete Springeth [u. a.] [Hgg.]: Gesammelte Schriften zur Literaturwissenschaft, Bd. 2: Lyrik II, Epik, Autobiographie des Mittelalters, Göppingen 2010 [GAG 750 II], S. 423-446, hier S. 424). Ihr fehlen »- vom Dargestellten her gesehen - die geschehene Tatsächlichkeit des Dargestellten (die zumindest behauptet wird), sie berichte[t] Fiktives; weiter fehlen der Biographie und der Er-Erzählung - vom Erzähler her gesehen - die Identität von erzählendem Subjekt und Erzählobjekt; nur bei der Autobiographie sind Autor und Erzähler einerseits (= Erzählsubjekte) und Erzählobjekt andererseits identisch« (ebd., S. 425). 
werden. »Diese Bruchstücke einer Beichtiger-Biographie enthalten [...] typische Umstände und Lebenssituationen eines wirklichen Beichtigerlebens in der Reformzeit: die Angabe, daß Beichtvater und Tochter noch leben, soll wohl das Lebensmodell bestätigen. « ${ }^{17}$ Durch »Zerlegung seelischer Gesamtzustände und Prozesse in mehr oder minder viele ১Stufen〈, ১Stationen oder >Schritte « ${ }^{18}$ orientiert sich die GR zudem, ohne selbst mystagogisch zu sein, an einer Grundform mystischer Autobiographie, die sich zur Entwicklungsgeschichte ausgebildet hat. ${ }^{19}$

Bereits der Prolog der GR $\left(\mathrm{Bl} .77^{\mathrm{r}}-80^{\mathrm{v}}\right)$ bietet eine komprimierte Darstellung der folgenden Beichtiger-Biographie, vermischt mit knappen Informationen über die Entstehungsgeschichte des Traktats und einer Inhaltsübersicht. Der zweite Abschnitt entspricht interessanterweise der Kurzfassung der >guldin regelı, wie sie drei Handschriften des 15. Jahrhunderts eigenständig überliefern (siehe S. 649):

Also nach disen vnderschaiden ${ }^{20}$ ist komen ain frowe, ain gaistlich person, vnd hat begert, das man ir ain gaistlich red herus mache. Vnd das sol genant sin ain sguldin regel mit siben capiteln. Vnd ist die materie, das die person kam zů irem bichtuatter vnd fraget nach dem ersten gebott vnd fiel ettwas red vff sy baide, das er sich der welte gancz vndezoch jn ainen wald. Si folget jm nach über sin willen vnd erfragte das ander gebott. Er zoch zů schůle vnd ward ain maister der hailigen geschrift. Si erfragte aber das dritt gebott. Darnach hielt er sich von vssen also behütsamlich, er erberklich ${ }^{21}$, wenn er hett nun wol der jar vnd getruwete jm gar wol, das sy mocht zů jm komen, wenn sy wolt. Vnd sie wolt vnd si erfragt die fierden fraug. Darnach kert er sich jnnerlich zů sinem grund vnd herczen vnd begund, fast vnd vil messe lesen vnd iederman vnhaimlich sin. Da erfragt sy das füft gebott. Darnach viel vff in kumer von des ordens wegen, das er warb vmb ain bistum, wie wol er es nit erfolget ${ }^{22}$. Da erfraugte si von jm das sechst gebott. Darnach begund er alten fast vnd krank werden jn dem ellende vnd starb vnd sie erfragte jn das sibend gebott jm leben vnd jm tod. Das ist die materie der 'guldin regel.

17 Ruh [Anm. 1], Sp. 393.

18 Misch [Anm. 19], S. 105.

19 Mit der >Vita nuova (um 1300) der Angela von Foligno (1248-1309), dem Rulman Merswin (1307-1382) zugeschriebenen `Büchlein von den Vier Jahren des anfangenden Lebens` und dem ¿Zweimannenbuch` des Gottesfreundes vom Oberland nennt Georg Misch Beispiele für diese Grundform mystischer Selbstbiographie (ders.: Geschichte der Autobiographie, Vierter Band, Erste Hälfte, Dritter Teil: Das Hochmittelalter in der Vollendung. Aus dem Nachlaß hg. v. Leo Delfoss, Frankfurt/Main 1967, S. 105-112).

20 Gemeint ist die auf S. 626 f. transkribierte Auflistung der sieben Arten von Geboten.

21 Frnhd. erwergklich, adj. >ehrbar, sittlich, züchtig`, siehe Christa Baufeld: Kleines frühneuhochdeutsches Wörterbuch. Lexik aus Dichtung und Fachliteratur des Frühneuhochdeutschen, Tübingen 1996, S. 63a.

22 Frnhd. erfolgen, v. >zustande bringen, erwirken`, siehe Alfred Götze: Frühneuhochdeutsches Glossar, Berlin 1967 (Kleine Texte für Vorlesungen und Übungen 101), S. 67a. Zu dieser Textstelle siehe S. 644, Anm. 52. 
Zi̊ dem ersten fragte sy iren bichtuatter: `Was sol ich tůn, das ich ingang in das leben? Er sprach: ‘Du solt dik bichten luterlichen. Z Z bessers wåre. Er sprach: 'Ja, fürbasser vor sünden vnd vor bichten hüte dich. Zu dem dritten: Si fragte jn, ain maister, ob bessers wåre. Er sprach: `Ia, in tugenden halte dich ordenlich.`Z Z dem fierden: Si fragte jm siner vswendigen gelaussenhait vnd miltikait, ob üczit bessers [Prädikat fehlt]. Er sprach: 'Ja, ain gůt bild der welt vortragen, besserlich vnd vnstrauffberlich, vnd dik vnd vil zů dem sacrament gaun. Zů dem fünften: Si fragte jn, ob üzit besser wäre, do si an jm sach, das er so gar jngezogen was vnd alle tag messe las. Si fragte jn, ob üczit bessers wäre. Er sprach: 'Ja, das ist dik vnd vil sich beraiten zů dem hailigen sacrament. Also solte man alle tag darzu gan vnd halten sich von jnnen fridlich. Zů sechsten fragte si jn, do sy sach, das er vsswendig jn kumer was, ob nit u்czit bessers wäre. Er sprach: `Ja, dik vnd vil in die badstuben gaun mit Cristo vff den ölberg vnd da herus schwiczen den aignen willen willenclich..$^{23} Z$ Z dem sibenden fragte si in jn siner krankhait vnd nach dem tod, ob üczet bessers wäre wenn das vorder gezalt. Er sprach: $>I$, durch den hag ${ }^{24}$ vnd durch den tzun vnd hurst ${ }^{25}$ brechen, so kumpt man in den garten des himelrichs. Des ist ain alte gewonhait, das man durch vil stechends lidens můss komen jn das himelrich. Also brach Ihesus durch den zoun des lidens vnd kam also in die glorie sins vatters. Er brach hin durch mit blossen henden vnd füssen vnd libes vnd haut vns den wege gemachet (Bl. $\left.78^{\mathrm{r}}-80^{\mathrm{v}}\right)$.

23 Die GR greift hier die Allegorie von der geistlichen Badstube auf, die andernorts selbstständig überliefert ist. So enthält Berlin, Staatsbibliothek, Ms. germ. qu. 405, Bl. $62^{\mathrm{r}}-65^{\mathrm{v}}$ (um 1500, bair.) unikal die >Geistliche Badstube` (ed. André Schnyder: Die geistliche Padstube. Eine spätmittelalterliche Andachtsübung, in: ZfdA 113 [1984], S. 146-157), die, »Bestandteil eines anonymen Traktats über die Straßburger Ursulabruderschaft « (ders.: [Art.] Die geistliche Badstube, in: ${ }^{2}$ VL, Bd. 11, 2004, Sp. 503), jedem Bau- und Einrichtungselement der Badstube (Boden, Wände, Decke, Ofen, Wanne, Röhren usw.) und den darin ablaufenden Vorgängen (Schwitzen, Reinigen, Ruhen, Schröpfen) eine Andachtsleistung in Form von Gebetsbeiträgen zuweist. Auch die Verbindung zwischen Schwitzen und Ölberg taucht in diesem allegorischen Traktat auf, allein die Verknüpfung mit dem Abtöten des eigenen Willens erscheint hier nicht: Item für das schwitzen zu ere dem, der des ersten geschwitzt hatt an dem ölperg, da er anfieng das pad der sel zu zerichten, vnd der selben angst vnd nott zu lob vnd dancksagung $v c$ veni gethan, yede mit $v$ Pater noster vnd Aue Maria (ed. Schnyder, S. 148, Z. 66-69). Kümper führt mit `Seelenbad und Geistliche Wirtschaft` (Karlsruhe, Landesbibliothek, Cod. Lichtenthal 103, Bl. 14 ${ }^{\mathrm{v}}-23^{\mathrm{v}}$ [1583]) ein weiteres, wohl im benediktinischen Umfeld Südwestdeutschlands entstandenes Beispiel für die Ausgestaltung der Badstubenallegorie an, die ebenfalls Bildelemente mit Gebetsanweisungen verknüpft, so auch das schwaisbad an den ölberg: Nem yetzundt die seln vnnd fuere syn in das schwaisbad an den ölberg. Bit den herren, das er seinen bluetigen schwais, die grose angst vnnd noth, schandt vnnd schmach, die er an dem ölberg gehabt, sambt anderm leiden vber die armen seelen gnedigklich well ausgüessen vnnd sy von allen sünden, schulden vnnd peinen weschen (ed. Hiram Kümper: `Seelenbad` und `Geistliche Wirtschaft`. Noch einmal zur Allegorie der Badstube in spätmittelalterlichen Andachtsübungen, in: ZfdA 139 [2010], S. 87-99, hier S. 94). Thomas Murners (1475-1537) >Badenfahrt ` von 1514 (ed. Victor Michels [Hg.]: Thomas Murner. Badenfahrt, Berlin u. Leipzig 1927 [Thomas Murners Deutsche Schriften mit den Holzschnitten der Erstdrucke I.2]) stellt im 33. Kapitel (`Das schweiß Bad`) keinen Bezug zum Ölberg her und verbindet das Schwitzen vielmehr mit der Beichte.

24 Frnhd. hag, m. `Einhegung, Gebüsch, Wald, eingehegter Ort‘, siehe Götze [Anm. 22], S. 114 a. 25 Frnhd. hurst, m./f. (alem.) >Dickicht, Hecke`, siehe Götze [Anm. 22], S. 127a. 
Demnach wendet sich die Beichttochter, hier als ain gaistlich person bezeichnet, mit der Bitte an eine unpersönlich genannte Instanz oder Person (man), ihr einen Lehrdialog, ain gaistlich red, über die sieben Arten von Geboten zu verfassen: >Die guldin regel in sieben Kapiteln; es ist dies freilich die toposhafte Legitimierung für ein geistliches Werk in der Volkssprache, dessen Abfassung vorgeblich von außen her in Auftrag gegeben wird. Es handelt sich nicht um die Verschriftlichung eines tatsächlichen Lehrgesprächs, sondern um einen fiktiven Dialog als ansehnliche und didaktische Verpackung der darin vermittelten Lehre, in dem die sauftraggebender gaistlich person freilich die Rolle der educanda übernimmt. Weder Beichttochter noch -vater, ein prediger maister (Bl. 121 ${ }^{\mathrm{v}}$ ), das heißt, Dominikaner, werden namentlich genannt. Die nach geistlicher Unterrichtung verlangende Beichttochter gibt in jedem der sieben Kapitel bzw. an jeder Lebensstation ihres Beichtigers den auslösenden Impuls für die interviewartig vermittelte Lehre, und zwar mit einer stets wiederkehrenden Einleitungsfloskel, mit der sie sich nach einer besseren Alternative für die im vorausgegangenen Kapitel vermittelte Verhaltensregel erkundigt, so etwa ১Sag mir an, lieber getrüwer vater, ist verzit bessers zů dem ingang des lebens wen dick vnd vil luterlichen bichten vnd dem gebot der natur gnůg tůn?`(Bl. 95 ${ }^{\text {r-v }}$; siehe auch den zweiten Abschnitt des Prologs auf S. 631). Lange Redepassagen in Respons auf die töchterlichen Fragen und die Auswahl dessen, was er ıseiner Tochter vermittelt, sind freilich dem Beichtiger vorbehalten. Bisweilen nimmt er es sich heraus, die töchterliche Neugierde in Schranken zu weisen (Bl. 82 $\left.{ }^{r-v}\right)^{26}$ oder die Beantwortung einer Frage kurzerhand abzuwürgen: >[...] vnd frag mich nit da von mer (Bl. 126 ${ }^{\mathrm{r}}$ ). Die Beichttochter ihrerseits schilt den geistlichen Vater mitunter des Versäumnisses wegen, habe er sie doch nicht rechtzeitig über einen bestimmten Sachverhalt unterrichtet, ${ }^{27}$ beklagt sich darüber, dass die väterliche Lehre ihr Begriffsvermögen übersteige und keine frömmigkeitspraktische Relevanz besitze - >Dise ding sint über min vernunft. Es gehoret in die schůl für die gelerten $\left(\mathrm{Bl} .119^{\mathrm{r}}\right)-$, oder muss den praeceptor sogar dazu ermahnen, seine Pflichten als geistlicher Lehrer trotz Zeitmangels (siehe 6. Kapitel, S. 644) und körperlicher Schwäche (siehe 7. Kapitel, S. 640) nicht zu vernachlässigen. All dies sind Äußerungen, die ein wohl männlicher Verfasser aus Sicht des Seelsorgers einer weiblichen Identifikationsfigur für

26 Lieber vatter, jn welhen gesaczten ist die cristanhait ob der alten iudischhait?^ Der vatter sprach: ১Was hast du ze fragen, das dich nit anegaut! Du soltest bichten luterlich vnd darus fragen. Vnd was nit zů der bicht gehört, das soltest du den gelerten befelhen $\left(\mathrm{Bl} .82^{\mathrm{r}-\mathrm{v}}\right)$.

27 , Lieber vater, wie zuchst du mich vmb mit den oren? Wa haust du dise lere so lang vor mir verborgen? Het ich sy langest gewist, ich wer ain ander mensch. Ich wand, es wer alles von vsnen genůg, die gebot behalten, als die juden thưnd` (Bl. 99²). 
simplices, die den Adressatenkreis der GR stellen, in den Mund legt und die fest zur literarischen Ausgestaltung der Lehrer-Schülerin-Beziehung gehören.

Bereits das 1. Kapitel (Bl. $\left.80^{\mathrm{v}}-94^{\mathrm{v}}\right)$ der GR macht klar, dass die intime Beziehung von >Vater` und >Tochter` unter den argwöhnischen Blicken der weniger bevorzugten Beichtkinder schon bald Ziel übler Nachrede wird: Im Rahmen von Belehrungen über das Beichtsakrament erkundigt sich die Beichttochter auch nach der jedoch für sie verneinten Möglichkeit, durch ainen botten [...] oder durch brief zu beichten, da der Beichtvater wohl wisse, das ich nit gar vil getar zu dir kumen von red wegen, die vff vns gefallen ist $\left(\mathrm{Bl} .91^{\mathrm{r}-\mathrm{v}}\right)$. Es bleibt unausgesprochen, ob dieser Argwohn seine Berechtigung hat, doch überschreitet die Tochter mit ihrer Sehnsucht nach Nähe zu sihrem`geistlichen Vater an einem Ort, an dem ihre Vertrautheit kein Ärgernis für andere darstelle (Bl. 94 ${ }^{\mathrm{r}}$ ), offensichtlich das übliche Maß. Die Vita der Apostolin Thekla dient ihr als biographische Schablone, um die eigene Gefühlslage zum Ausdruck zu bringen und folglich die eigene Situation auf das gleichermaßen intime Verhältnis zwischen Apostel und Apostolin zu beziehen:

Sy sprache: ‘Lieber getrüwer vater, wiltu, so volg ich dir noch. Ich will da hin, da niemen an dir vnd an mir nit geergert wirt. Ich wil got haimlich leren werden vnd allenn menschen vnhaimlich in ainem wald oder in ain arm closter, da wenig brüder inne sint. « Sy sprach: >Ich volg dir nauch als Teckla sant Paulus, wann ich diner lere nie baß bedorft wan hüte diß tages. Lieber vater, got behut dich vnd dank im selber, der brun aler tugent, aller diner trüwe vnd gůtter lere, so ich von got vnd von dir entpfangen hab. Vnd vieng an inecklich ze wainen vnd gieng von im betrupt, das vil ander bichtkint geergert wurden, die das sahen, vnd fielen vil wort vff sy baide. Da er frů meß laß, do furr er enweg vnd set niemen nit. Sy schrye vnd waint vnd waz betrübet, wen es was alles mit natur vermenget, das sy da sůchet, vnd es wart gnad her nach dar $v \beta$, wan sy ließ nit abe, sy enkeme den vff die höche ains volkomnen menschen vnd hieng im nauch, biß das er starbe vnd sy bald nach im (Bl. $\left.94^{\mathrm{r}-\mathrm{v}}\right)$.

Ihre übermäßige Anhänglichkeit zwingt den Beichtiger am Ende des 1. Kapitels zum heimlichen Rückzug in den wald (siehe Prolog) und bringt ihr, die sie mit ihrem tränenreichen Abschied erneut Ärgernis unter den übrigen Beichtkindern, vielleicht der Gemeinschaft eines Dominikanerinnenklosters (siehe S. 641), erregt, den Vorwurf ein, ihre Sehnsucht sei mit natur vermenget. Selbst der Beichtvater lässt von Selbstzweifeln an der eigenen Standhaftigkeit erst in höherem Alter ab, wenn er im 3. Kapitel endlich davon sprechen kann, er hett nun wol der jar vnd getruwete jm gar wol, das sy mocht zů jm komen, wenn sy wolt (Prolog, Bl. 79' $)$.

Mit Übergang vom 1. zum 2. Kapitel $\left(\mathrm{Bl} .95^{\mathrm{r}}-110^{\mathrm{r}}\right.$ ) beginnt ein sich über den gesamten Traktat erstreckendes Wechselspiel von Trennung und Wiedersehen, 
das der Legende der Thekla von Ikonium ${ }^{28}$, Protomärtyrerin des 2. Jahrhunderts, nachempfunden ist. Ihre Vita sei hier knapp zusammengefasst:

Die Heidin Thekla wohnt der Makarismenpredigt bei, die der Apostel Paulus (vgl. Act 13,50) im Haus des Onesiphorus in Ikonium hält, und bekehrt sich daraufhin zum Christentum; drei Tage und Nächte hört sie ihm vom Fenster ihres Hauses aus ununterbrochen zu. Ein Eheleben mit dem Verlobten Thamyris ist für sie nicht mehr vorstellbar, der, eifersüchtig und erzürnt, die Ikonier gegen Paulus aufwiegelt, da er die jungen Männer und Frauen zu einem Leben in Keuschheit und Ehelosigkeit verführe. Heimlich besucht Thekla den in Haft genommenen Apostel im Gefängnis, um die ganze Nacht hindurch seinen Belehrungen zu lauschen. Am folgenden Morgen entdeckt, wird Paulus aus der Stadt vertrieben, an Thekla hingegen ein Exempel statuiert: Ihr droht der Feuertod, dem sie jedoch durch göttliche Hilfe entflieht. Thekla, die sihren Apostel nach längerer Suche wiederfindet, möchte ihn fortan auf allen seinen Wegen begleiten. Der zunächst zögerliche Paulus begibt sich schließlich mit Thekla nach Antiochien, wo der Syrer Alexander vergebens um sie wirbt. Als Rache für Theklas demütigende Abweisung denunziert sie dieser und erreicht eine Verurteilung zum Tierkampf. Diesen übersteht Thekla auf wundersame Weise unverletzt und vollzieht an sich die Selbsttaufe. Anschließend folgt Thekla in Männertracht Paulus nach Myra und erhält vom Apostel den Auftrag, Ikonium zu missionieren. In Seleukia verbreitet sie später das Wort Gottes und stirbt dort eines natürlichen Todes. ${ }^{29}$

Tatsächlich gelingt es der Beichttochter, ihren Beichtiger nach seinem Fortgang, obgleich über sin willen (siehe Prolog, S. 630), aufzuspüren und sich ihm, ganz wie einst Thekla dem Paulus, verkleidet und daher zunächst unerkannt zu nähern:

28 Die Legende befindet sich zuerst in den Paulusakten (185-195), neben den Akten des Philippus, Thomas und Andreas, einer der apokryphen Schriften, "qui font apparaître des femmes aux côtés des Apôtres, fiancées ou mariées, que poursuivent leurs fiancés ou maris, tantôt désespérés tantôt furieux qu'elles se refusent à eux après s’être faites chrétiennes « (Gilbert Dagron: Vie et miracles de sainte Thècle. Texte grec, traduction et commentaire, Brüssel 1978 [Subsidia Hagiographica 62], S. 35). Seit Tertullian († 220/240), welcher der Ämtervollmacht von Frauen in Anlehnung an Thekla ablehnend gegenübersteht, werden dogmatische Bedenken gegen die >Acta Pauli et Theclae` erhoben. Hieronymus zählt sie zu den Apokryphen und das >Decretum Gelasianum` verwirft sie (siehe Klaus-Gunther Wesseling: [Art.] Thekla, in: Friedrich Wilhelm Bautz [Hg.]: Biographisch-Bibliographisches Kirchenlexikon, Bd. 11, 1996, Sp. 806-814). 29 Vgl. Léon Vouaux: Les actes de Paul et ses lettres apocryphes. Introduction, textes, traduction et commentaire, Paris 1913 (Les apocryphes du nouveau testament 3), S. 1-12; Wesseling [Anm. 28], Sp. 806 sowie Martin Ebner u. Markus Lau: Überlieferung, Gliederung und Komposition/Übersetzung der Theklaakten, in: Martin Ebner (Hg.): Aus Liebe zu Paulus? Die Akte Thekla neu aufgerollt, Stuttgart 2005 (Stuttgarter Bibelstudien 206), S. 1-29, hier S. 5-11. 
Die bichtochter macht sich vff vnd erfraget, wo ir bichtvater was, vnd kam zů im in ainen gaistlichen schin, das er ir nit bekannt. Sy sprach: ১Here, ich bin üwerr kind. Bicht hant ir mitt gott für mich beten.`Sy sprach: `Ich han got gebeten, das ich in minem herczen din vnd aller creature vergessen.« Sy sprach: `Ich bin reht aber hie, ich wil dich můde fragen.« Er sprach: 'Der wille gotes werder (Bl. 95 ${ }^{\mathrm{r}}$ ).

Und auch wenn der Beichtvater seine Tochter wie hier letztlich immer wieder gewähren lässt, beklagt er sich im Zusammenhang mit Ausführungen zum Gebot der Vater- und Mutterliebe durchaus über die Mühen, die sie und die übrigen Beichtkinder ihm als geistlichem Vater bereitet haben:

\begin{abstract}
Liebe thochter, was hab ich der hundesflügen ${ }^{30}$ gehabt von dir vnd von andren bichtkinden! Was lid ich snawe $e^{31}$ vnd schmachait! Mir tete doch nieman wierser wen mine bichtkind, do die red vff dich vnd mich viel. Ich kund mich der flügen kum erweren, wen das ich gedaucht, sy fliegent bald. Die welt die kan nit die lenge vff ainer red beliben. Wer der welt der ougen zů, tůt ir ouch denn mund zủ. ${ }^{32}$ Bin ich nun die hundesflügen geflochen, so komest du aber mir noch. Aber fürcht ich nit hundesflügen vnd machst mir ein red vff ain ermel. Stand vff vnd gang haim! ‘ Do fiel sy im zů fůsen vnd begert, das er sy liese beliben an sinen schaden. Er erbarmet sich über sy $\left(\mathrm{Bl} .100^{\mathrm{r}-\mathrm{v}}\right)$.
\end{abstract}

Das 2. Kapitel $\left(\mathrm{Bl} .95^{\mathrm{r}}-110^{\mathrm{r}}\right.$ ) über den Dekalog und die zehn, als Strafen für den Verstoß gegen die Gebote gedeuteten Plagen der Ägypter (Ex 7,14-11,10) >korrigiert ` das erste insofern, als es nun nicht mehr allein darum geht, die einmal begangenen Sünden möglichst häufig sabzubeichten`, sondern daz man sich vor sunden hüte vnd ouch vor nüwer bicht $\left(\mathrm{Bl} .95^{\mathrm{V}}\right)$, das heißt, die Sünde gar nicht erst zulässt, sodass Beichte nicht zur routinemäßigen Sündenwaschung degradiert. Mit seinem Bestreben, auch eigenen Verfehlungen vorzubeugen und sich den auslösenden Momenten der eigenen Sündhaftigkeit - hier die Welt bzw. der enge Kontakt zur Beichttochter - nach dem Vorbild Johannes' des Täufers zu entziehen, rechtfertigt er ihr gegenüber sein plötzliches Verschwinden im 1. Kapitel:

Der vater: `Es ist vast beser, das man halt die zehen gebot gotes me gaistliche wen zitlich vnd daz man sich vor sunden hüte vnd ouch vor nüwer bicht. Daz ist besser, wer gar wol gebichtet. Darvmb bin ich hergeflogen von der menge, das ich mich hüte, das ich nit zů bichten hab. Also floch in denn wald Iohanes baptista (vgl. Lc 1,80), das er sin leben nit beflecte ioch mit täglichen sünden, vor den er nit behüet was noch gehailget in můter lip $\left(\mathrm{Bl} .95^{\mathrm{v}}-96^{\mathrm{r}}\right)$.

30 Frnhd. hundsvlieg, f. >Hundsmücke, Schnake`, siehe Baufeld [Anm. 21], S. 133a; die vierte der zehn ägyptischen Plagen (Ex 8,16-31).

31 Mhd. snabe, stswf. `Mangel<, siehe Matthias Lexer: Mittelhochdeutsches Handwörterbuch, Bd. 2, 1876, Sp. 1021.

32 Bei dieser syntaktischen Konstruktion handelt es sich um ein Apokoinu; zu ergänzen wäre daher ein weiteres Prädikat tůt im vorangehenden Subjektsatz. 
Das Kapitel schließt mit Ausführungen zu den Anfechtungen und den geistlichen Waffen (siehe unten und S. 643) und gibt am Ende einen Ausblick auf die unmittelbaren Zukunftspläne des Beichtigers, das Theologiestudium in der Fremde. Dies bedeutet für die Tochter eine viele Jahre währende, unüberbrückbare Trennung. Über (Send-)Briefe hält der geistliche Vater jedoch den Kontakt mit ihr aufrecht:

Er sprach: ১Du hast got, den ruff an. Ich wil zů schůl faren in frömde lannd.`Sy gehůb sich übel, er schiede von ir in frömde land, dar sy nicht komen möcht, vnd belib lang $v \beta$, biß das er maister der hailgen geschrifft ward. Da zwischen schribe er ir mengen brieff, das sy fest belib by siner lere. Das tet sy vnd wart fast ain ernsthaffter, lieber mensch. Amen $\left(\mathrm{Bl} .110^{\mathrm{r}}\right)$.

Im 3. Kapitel $\left(\mathrm{Bl} .110^{\mathrm{v}}-120^{\mathrm{r}}\right)$ nach vil iaren wider vmb von schůl heimgekehrt, nimmt der Beichtvater, nun maister der hailgen geschrifft, eine rege Predigttätigkeit auf. In seinen Predigten lehrt er, wie man tugent üben sol nach dem mitel vnd mauß (Bl. 110v), das heißt, nach oberster Maßgabe der Liebe, wan on die sint die tugent schwach vnd krank vnd formlo $\beta\left(\mathrm{Bl} .112^{\mathrm{r}}\right)$. Die geistliche Tochter, die von da an wieder in seiner Nähe ist, erkennt er erst an der für sie typischen Fragefloskel, in die sie diesmal auch eine Rekapitulation der im 2. Kapitel gelehrten, geistlichen Waffen mischt:

\begin{abstract}
Die thochter kam zů im, do bekant ers nit. Sy fragte ine: 'Lieber here vnd maister, wz ist besser czů dem ingange des lebens vnd dick vnd vil luterlichen bichten vnd sich vor sunden behüten vnd die zehen gebot gaistlich halten? « Do bekant er sy in der frag vnd sprach: >Thochter, lebest du noch? Sy sprach: Ja, laider ia, das ich nit erhencket ward, ertrenket, erstochen, verbrant, lebendig begraben, do ich och ye haimlich wart.، Er sprach, wie sy das mainte, er het sy das böst gelertt. Sy sprach: 'Ich niem das crütz Ihesus, do er selber anhieng. Ich main das sper, do er mit gestochen wart. Ich main sin kostbar plüt, do menig lieper mensch in ertrincket vnd entsencket. Ich main der minen brant vnd sin hailges grap. Dis waffenclaid gabend ir mir zů letz, do ire zů schůl füren. Ich fraugen dich wider, maister, vmb die dry fraug` $\left(\mathrm{Bl} .110^{\mathrm{v}}-111^{\mathrm{v}}\right)$.
\end{abstract}

Das 4. Kapitel (Bl. $120^{\mathrm{v}}-126^{\mathrm{r}}$ ) verdeutlicht, dass die Lehren des Beichtvaters keine leeren Worte sind, sondern dass er sie in Übereinstimmung von vita und verba vorlebt; ein ganzes Jahr über ist er seinem Umfeld ein wahrer spiegel aller zucht:

Der maister fieng an so sitlichen vnd so behůtsamlichen leben vor den lüten vnd allain, vnd warent die geberd so geordnet vnd mit züchten gezieret, des er vil menschen in sin bicht zoch. Er ward ain spiegel aller zucht. Also ordnet er den vßwendigen menschen, von dem wir ouch gezügnuß müssent haben, als sant Pauls spricht (vgl. I Cor 6,12-20). Er lertz ouch vil menschen an allen predigen, die er tet ain gantz iar, vnd das sunst in der bicht dick vnd vil zu dem hailgen sacrament gan, vnd was milt siner wort vnd siner werck gegen armen menschen $\left(\mathrm{Bl} .120^{\mathrm{v}}-121^{\mathrm{r}}\right)$.

Der neue fromme Ratschlag, den der Beichtiger erteilt, ist häufige Kommunion; dieses Kapitel gibt daher zahlreiche Belehrungen über den Empfang des Sakraments, das [...] ist gnad vnd bringet gnäd vnd meret gnäd (Bl. 122 ${ }^{\mathrm{r}}$ ). Sein vorbildlicher Lebenswandel, der seinen Lehren besondere Heilswirksamkeit verleiht, 
führt freilich dazu, dass der Beichtvater unfreiwillig geistlichen >Ruhm Gläubigen erwirbt. Auch die Beichttochter möchte, im Genuss dieser Lehren, von seiner heilsmäßigen >Ausstrahlung` profitieren:

Des kam aber die bichttochter vnd sprach: >Alle stat růnt üch an uẅern wisen vnd werdent vil lüt an uch gebesert. Dz bin ich gar fro.« Er sprach: 'Es sprichet Senica, es ist vil beser Roms wirdig sin, wen gar woll gerůmet werden.く Die tochter sprach: sI sint iederman milt. Des erzögent ouch an mir vnd saget, ist ietzunt besser wen dick bichten, biterlich sich vor sünden hüten sunderlichen vnd sich in tugent üben flißclich? [...] Der prediger maister sprach: 'Ja, das ist vil bösser, das man dick vnd vil in dem iar zů dem vilhailgen sacrament gang. Wenn der mensch, der gebichtet hat vnd sich vor sünden gehüt haut vnd sich in tugenden geübt haut, der haut nun kain hindernuß me, wen das er enpfauch vil vnd dick das sacraments (Bl. $\left.121^{\mathrm{r}-\mathrm{v}}\right)$.

Das 5. Kapitel (Bl. $\left.126^{\mathrm{r}}-133^{\mathrm{v}}\right)$ beschreibt einen folgenreichen Wandel in der geistlichen Entwicklung des Beichtvaters, zieht er sich, der zuvor öffentlich gelehrt hat, doch plötzlich in sin conscientz zurück vnd ward allen menschen vnhaimlich vnd nam sines hertzen war vnd siner conscientz. Er laß menig meß vnd hort wenig bicht vnd brediget nit vil. Es nam die lüt wunder, das er aber an sich ain nüwe wiß hett genomen (Bl. $126^{\mathrm{r}-\mathrm{v}}$ ). Dieser Rückzug in die Innerlichkeit wirkt wie eine Revision des bislang nach außen gerichteten Wirkens. Analog zum Übergang vom 1. (Beichthäufigkeit) zum 2. Kapitel (Vermeidung von Sünde) erfolgt auch hier eine >Korrekturs: Die bloße Häufigkeit der Kommunion als Richtschnur für ein gottgefälliges Leben wird um das sich selber beraiten zů dem sacrament verfeinert. Denn swer nit alle tag berait ist, do ist zů fürchten, das er ouch einest in dem jar nymer recht berait werde. Dar vmb, liebe tochter, berait dich alle tag, als solte man dich des tages in ain grab legen $\left(\mathrm{Bl} .133^{\mathrm{r}-\mathrm{v}}\right)$. Den pflichtgemäßen Sakramentsempfang hat die vom einzelnen Kommunionstermin unabhängige, fortwährende und im vollen Bewusstsein der Kürze menschlichen Lebens stehende Selbst- bzw. Gewissensbetrachtung vorauszugehen (nah flißigem ersưchen der conscientz vnd sich doch für ainen sünder schetze[n], $\left.\mathrm{Bl} .133^{\mathrm{r}}\right)$ :

Des kam die tochter aber dar vnd sprach: 'Sagent an, maister, sind ir zů ainem stumen worden? Was hand ir nun vor? Sagent an, was ist besser wen dick vnd vil zů dem sacrament gän, das ich ingang in das leben.< Der maister sprach: >Das ist besser, das du dich beraitest zů dem sacrament alle tage vnd stund, als wer es gewonhait der cristenhait alle tag xxiiij zůzegand. Dar zů werest du berait. Also tett Maria Magdalena. Die beraitt sich xl iar, das sy an irem end mocht enpfauchen daz sacrament. ${ }^{33}$ Also halt ich mich jetzunden ine, wen ich

33 Siehe Jacobus de Voragine: >Legenda aurea<, cap. XCVI (ed. Theodor Graesse [Hg.]: Jacobi a Voragine Legenda aurea. Vulgo historia Lombardica dicta, Breslau 1890 [ND Osnabrück 1969], S. 413). 
lernen, meß leßen vnd berait mich zů der mesen. Dar vmb beraitschaft zů dem sacrament ist besser wen das sacrament enpfauchen vnberait. Doch ist das sacrament besser mit beraittschaft enpfauchen, als der tauff des gaistes vnd das wasser besser ist wen allain des gaistes, so ist es nützer zů beserung dem menschen. So ist es got ain grose ere, geware diemůt, volkomen vorcht, luter andaucht vnd gotes wirdigkait. Das alles vollget, ob man das sacrament berait entphauchets $\left(\mathrm{Bl} .126^{\mathrm{v}}-127^{\mathrm{v}}\right)$.

Der Beichtvater gibt seiner geistlichen Tochter daraufhin Anweisungen für die beraitschaft zur Kommunion und muss ihr (bis Bl. 132 $2^{\mathrm{v}}$ ) auch ob ihrer geistlichen scrupulositas, sie könne eventuell nicht ausreichend, das heißt, im Stand der Todsünde verbleibend, auf den Sakramentsempfang vorbereitet sein, Linderung verschaffen.

Ein herber Einschnitt (nit clain liden) im Leben des Beichtvaters, der eine tragische Verkettung äußeren Leides mit sich bringt, steht am Beginn des 6. Kapitels (Bl. $134^{\mathrm{r}}-141^{\mathrm{r}}$ ): Er wird aus dem eigenen Konvent ausgestoßen und stellt sich - der Prolog spricht hingegen von der Bewerbung um ein bistum $!^{34}-$ in den Dienst eines grosen heren vnd [...] siner frouen. In ihrem Auftrag begibt er sich als Bote zweimal zum Papst nach Rom und wird Opfer eines Raubüberfalls:

Dem maister viel zů nit clain liden, das er mit den ardren ${ }^{35}$ von sinem closter vertriben ward von groser wirdigkait nun sinen obren. Also kam er zů ainem grosen heren vnd zů siner frouen, die teten im grose ere an, vnd ward ir bichtvater vnd gaben im brieff an den baubste vnd für zway maul gen Rom in gesteinkesten vnd schůff ${ }^{36}$ nit vnd ward gefangen vnd ward in sin gelt genomen vnd kam in groß liden, schuld vnd armůt. Also gab er sich gedultigclich in das vser liden vnd gab im von inen nit zů schaffen (Bl. $134^{\mathrm{r}-\mathrm{v}}$ ).

34 Siehe dazu S. 644, Anm. 52. Es ist nicht mit Sicherheit zu klären, wie diese Diskrepanz zustande kommen konnte. Vielleicht liegt dieser Widerspruch in einem Vorlagen- oder Verfasserwechsel begründet. Immerhin wurde die GR von zwei Händen niedergeschrieben, der vordere Teil (Bl. $\left.77^{\mathrm{r}}-93^{\mathrm{r}}\right)$ mitsamt des Prologs von der zweiten, der hintere Teil (Bl. $93^{\mathrm{r}}-148^{\mathrm{v}}$ bzw. Bl. $153^{\mathrm{r}}$ ) mitsamt des 6. Kapitels von einer dritten Hand, die sich in Ms. Praed. 159 unterscheiden lassen (siehe Clemens Köttelwelsch [Hg.]: Katalog der Stadt- und Universitätsbibliothek Frankfurt am Main, Bd. 2: Die Handschriften der Stadt- und Universitätsbibliothek Frankfurt am Main. I. Die Handschriften des Dominikanerklosters und des Leonhardstifts in Frankfurt am Main. Beschrieben von Gerhard Powitz, Frankfurt/Main 1968, S. 353).

35 Frnhd. ordren - >Befehl, Order - mit Übergang von mhd. $o$ vor $r$ zu offenem $o$ bzw. $a$, wie ihn das Bairische schon früh kennt (siehe Virgil Moser: Frühneuhochdeutsche Grammatik, I. Band: Lautlehre. 1. Hälfte: Orthographie, Betonung, Stammsilbenvokale, Heidelberg 1929 [Germanische Bibliothek. Abt. 1, Sammlung germanischer Elementar- und Handbücher. Reihe 1, Grammatiken 17,1], S. 133 [§ 73 Anm. 1]); evtl. handelt sich dabei auch um eine Verschreibung von andren. In diesem Fall wäre der Beichtiger zusammen mit anderen Ordensbrüdern aus dem Konvent ausgestoßen worden.

36 Frnhd. schaffen, v. ıbewirken, ausrichten`, siehe Götze [Anm. 22], S. $184 a$. 
Dem äußeren Leid begegnet der Beichtvater mit geduldigem Ertragen, eine heilbringende Lebens- und Leidensstrategie, die er auch seiner Beichttochter vermitteln möchte: Besser als alles bisher Gesagte, und dies ist eine weitere Steigerung der Lehre und der inneren Entwicklung des Beichtvaters, ist das geduldige und gehorsame Ertragen äußeren, das heißt, nicht selbst gewählten, von anderen Menschen verursachten bzw. auferlegten (fremd liden) und somit von Gott verhängten Leides und, in gleichem Zuge, das Abtöten des Eigenwillens, der natürlicherweise gegen dieses unfreiwillig zu tragende Leid anzukämpfen versucht: swer dich nit lides, anderst du wirst lidens nimer lidig`(Bl. 136 v); das von außen auf den Menschen zukommende Leid korrespondiert hier mit dem ebenso von außen her, das heißt, heteronom gegebenen Gesetz, sei es weltlich oder kirchlich (6. Gebot der GR). Die Bekämpfung eigener Sündhaftigkeit allein (2. Kapitel) genügt nicht mehr. Mit diesem frommen Verhalten, das ihn erneut von seinem sozialen Umfeld entfernt und aufgrund dessen er sich gezwungen sieht, seinen Dienst für die Herrschaft aufzukündigen, stellt er sich in die Christusnachfolge. Zudem nennt er mit der heiligen Elisabeth von Thüringen ein weibliches, für die Beichttochter empfehlenswertes und aus der Sicht des Seelsorgers vielleicht auch besseres Vorbild für fromme Frauen als Thekla von Ikonium, in deren Nachfolge sich die Tochter ihrerseits stellt (1. Kapitel):

\begin{abstract}
Also ward er aber den luten vnhaimlich vnd fiel red vff in. Das halff im alles got tragen vnd schied sich von der herschaft vnd nam sich ainer ernsthafter wiß an. Vnd wen man in sücht, so hieß er sagen, er wer in die batstuben gegangen. Vnd das waz, war er gieng, mit Cristus vff den ölberg vnd schwitzet da her $v \beta$ sinen aigen willen. ${ }^{37}$ In den ziten do kam aber die tochter vnd claget in gar ser in sinem liden. Er lacht wol gütlich vnd sprach: sIch enkond hie nit ze liden komen, ich můst liden von Rom holen. Sy sprach: ‘Lieber vater vnd freund gotes, ist vczet besers wen beraitschaft zů dem hailgen sacrament? [...] Der maister sprach: `Ja, das ist besser, das der mensch gehorsam sy dem gebot des götlichen geschribnen rechten ine dem rechtbůch. Vnd das ist dick vnd vil in die bůchstaben gegangen vff den ölberg mit Cristo. Do er schwitzet, wz dar ser vnd blü von allen sinen gelidern, do schwitzet her $v \beta$ sinen aignen willen. Den gab er dem vatter. Also solt du hervsschwitzen dinen aigen willen. Vnd als Cristus gab sinen willen ine den willen sins vaters, also soltu geben dinen willen in den willen des sunes vnd solt lernen willen brechen vnd schwitzen, wie we es dir ti̊t. So sant Elisabett vnderstand, den vsetzigen die füß zů weschen, so satzt sy den kubel an den mund vnd trank dar $v \beta$, das ir natürlicher wil gantz erstarb $\left(\mathrm{Bl} .134^{\mathrm{v}}-136^{\mathrm{r}}\right) .^{38}$
\end{abstract}

$37 \mathrm{Zu}$ diesem Motiv siehe S. 631, Anm. 23.

38 Das Bild von der das Badewasser von Leprakranken trinkenden Elisabeth lässt sich etwa in serm. 109 der `Sermones de sanctis Biga salutis intitulati` (Druck: Hagenau 1499) des ungarischen Franziskaners Oswald von Lasko († 1511) finden: Contigit, ut balneum foetidum et horridum leprosi naturali horrore abhorreret, sed in se reversa dixit sibiipsi: "Quae es tu, peccatorum sceno deturpata, ut contemnas istius infirmi foetida vulnera?" Mox inaudito fervore utraque manu hauriens aquam 
Das 7. und damit letzte Kapitel $\left(\mathrm{Bl} .141^{\mathrm{r}}-148^{\mathrm{v}}\right)$ zeigt einen kränkelnden Beichtvater, der, im stüblin eines unbekannten Klosters zurückgezogen, dem Ende eines, im Sinne des 6. Kapitels leidvollen Lebens entgegensieht. In Erinnerung an eine Sentenz des Seneca ermahnt er sich jedoch dazu, seine übrige Lebenszeit in den Predigtdienst $\mathrm{zu}$ stellen und die Menschen, seiner momentanen Lebenssituation entsprechend, über das Sterben als das älteste Gebot der Welt (gebot gůter alter gewonhait) und über die Vorbereitung auf den Tod $\mathrm{zu}$ unterrichten. Gleiches verlangt die Beichttochter bei ihrer letzten Begegnung, die das Lehrgespräch ob seiner gesundheitlichen Angeschlagenheit regelrecht einfordern muss:

Do nun der maister sich fast erlaiten haut dis vnd daz, do begund er alten vnd sich werden von tag zů tage. Er hett im gern geholffen mit artznyen. Im ward in ainem closter ain stüblin. Darin belip er vnd ward der welt vnhaimlich vnd ouch sinen brüdern. Er gedaucht: ‘Du solt recht lernen schriben, wen Senica spricht, es ist vnmuglich, das ain alterer mensch lang leb, vnd ist aber gar müglich, das ain junger mensch bald sterb. Vnd so er prediget, so prediget er fast von sterben vnd von dem tod. Die bichttochter kam vnd sprauch: >Maister, was ist beser wen dick vnd vil baden ${ }^{39}$ vnd gehorsam sin den geboten der rechten vnd vfsetzung der hailgen kirchen? Der maister sprach: ‘Bistu aber hie, ich bin krank vnd mag mich nit mit dir bekimern.く Die tochter sprach: ,Du wilt doch lernen sterben, so ler mich leben, das ich ingang in das leben.r Der maister sprach: >Das gröst vnd das hindrost das ist dz gebot gưter alter gewonhait, das ist sterben. Die gewonhait hätt gewert wol sechstusent iar ${ }^{40}$. Dar vmb dz höchst ist: Durch zwy, durch den hag vnd durch die hurst ${ }^{41}$ so kumet man in den hochsten garten, wen der gart des himelriches vnd des ewigen lebens ist bezunet mit mengem stechenden liden vnd vmbhagt mit mengerlay sterbenn, dar durchbrechen můß, als Paulus spricht: ‘Durch vil betrübnuß müsen wir ingän in das rich der himek (Act 14,22)< (Bl. 141 ${ }^{\mathrm{r}}-142^{\mathrm{v}}$ ).

Im Anschluss an umfassende Belehrungen $\mathrm{zu}$ Vorbereitung und Nutzen des Sterbens bleibt dem Beichtvater nur noch, die Beichttochter um Gebete für sein Seelenheil zu bitten: svnd horest du minen tot, so bitt got für mich. Das will ich ouch für dich, vnd gebrist dir ietzunt zů fragen, so frag ein söl in dem fegfür. Die sol dich vil woll vnderwissen von ainem sterbenden menschen $\left(\mathrm{Bl} .148^{\mathrm{r}-\mathrm{v}}\right)$. Der kurze Epilog

balnei foetidi et insanie pleni ebibit, ut sic seipsam superaret. $O$ inauditus fervor pietatis, o ineffabilis Christi amor quid facit? Ecce hanc regis filiam in purpuram nutritam ancillis ancillam fecit (http:// sermones.elte.hu/szovegkiadasok/latinul/laskaiosvat/index.php?file=os/os109, Aufrufdatum: Oktober 2014). Ich danke Herrn Dr. des. Richard F. Fasching herzlich für diesen Hinweis.

$39 \mathrm{Zu}$ diesem Motiv siehe S. 631, Anm. 23.

40 Aus der Anzahl von sechs Schöpfungstagen (Gn 1,1-31) und Ps 89,4 (quoniam mille anni ante oculos tuos tamquam dies hesterna quae praeteriit et custodia in nocte) ergibt sich für die Welt nach mittelalterlicher Auffassung ein Weltenalter von 6000 Jahren.

41 Siehe S. 631, Anm. 24 und 25. 
(Bl. $148^{\mathrm{v}}$ ) gibt an, dass die GR am 19. Januar 1434 vollendet wurde. ${ }^{42}$ Beide, Beichtvater und -tochter, seien in jenem Jahr noch am Leben gewesen:

Die tochter seig nider vff ir knüe vnd dancket gott, der ir die rregel haut gegeben durch den maister, vnd ward die regek volbracht in dem jar, do man zalt $m^{\circ} c c c c^{\circ}$ xxxiiij ${ }^{\circ}$ iar vff dem xiij. tag der hailigen dri künigen. Do lept der maister noch vnd ouch die bichtochter. Gott geb in baiden sin götlich gnaud, nach disem leben ainen ingang in das ewig leben etc (Bl. 148 ${ }^{\mathrm{v}}$ ).

\section{III. (Pseudo-)Biographisches in der iguldin regels}

Im Gegensatz zum Beichtvater erfährt man über die Biographie der Tochter nichts Näheres. Die Vermutung, sie gehöre, da von einem prediger maister seelsorgerisch betreut, ebenfalls dem Dominikanerorden an, ${ }^{43}$ steht im Widerspruch $\mathrm{zu}$ ihrer offensichtlich uneingeschränkten Freizügigkeit. Sie erlaubt es ihr, den Beichtiger mehrfach aus eigenem Antrieb und auch über größere Distanzen hinweg aufzusuchen, ihm nachzureisen. Zudem nimmt sie, was scheinbar nicht ihrem Stand entspricht, einen gaistlichen schin an, um sich dem Beichtvater unerkannt zu nähern (2. Kapitel). Die >Kontamination` der Darstellungen von geistlicher Tochter und nicht monastischer Theklafigur muss jedoch zwangsläufig zu Widersprüchen führen, was Überschneidungen und Motiventlehnungen betrifft. Gemein ist ihnen jedoch das manisch anmutende, unstillbare Verlangen nach Nähe zum Apostel bzw. Beichtiger, das in den Augen der Umstehenden - den Ikoniern bzw. den übrigen Beichtkindern - das übliche Maß an Vertrautheit bei Weitem übersteigt. Beide Frauen übertreten dabei empfindliche Grenzen: Die Beichttochter bewegt sich am Rande dessen, was an intimer Vertrautheit zu einem Geistlichen schicklich ist, und Thekla entzieht sich darüber hinaus ihrer Rolle als Ehefrau. ${ }^{44}$ Mit der

42 In der Beschreibung von Ms. Praed. 159 durch Diederichs ist die Jahreszahl 1434 irrtümlicherweise als 1472 ausgezeichnet (siehe Ernst Diederichs: Meister Eckharts >Reden der Unterscheidung`. Eine literarkritische Untersuchung, Diss. Halle/Saale 1912, S. 4).

43 In observanten Konventen des Ordens übernahm stets ein Predigerbruder die Beichte der Nonnen; Klosterkapläne hielten zur Entlastung der Mönche lediglich die Konventmessen (siehe Marie-Luise Ehrenschwendtner: Die Bildung der Dominikanerinnen in Süddeutschland vom 13. bis 15. Jahrhundert, Stuttgart 2004 [Contubernium 60], S. 245 f. und S. 251).

44 Die Legende äußert durchaus Bedenken gegenüber Theklas Motiven für die sonderbare Beziehung zum Apostel: So finden die Ikonier die beiden im Gefängnis an, aneinander "gewissermaßen mitgefesselt durch ihre Liebe«, und kurz vor der gemeinsamen Abreise nach Antiochien äußert Paulus folgende Warnung: „Die Zeit ist böse, und du bist schön von Gestalt. Daß nur nicht eine andere Versuchung über dich komme, schlimmer als die erste [Thamyris, S. A.] und du nicht aushältst, sondern mannstoll wirst« (Edgar Hennecke [Hg.]: Neutestamentliche 
gemeinsamen Reise nach Antiochien und Theklas Missionsauftrag geht die Legende freilich sehr viel weiter als die GR. Ein historisches Vorbild für die Beichttochter ist bei all dem nicht auszumachen. Außergewöhnlich sanhänglicheく Beichtkinder, sei es inner- oder außerhalb des Klosters, und eine über das übliche Maß hinausgehende Vertrautheit mit Beichtvätern wird es im Mittelalter, obgleich in observanten Frauenklöstern Vorlieben für bestimmte Beichtiger nicht berücksichtigt werden sollten, zweifelsohne gegeben haben. ${ }^{45}$

»[F]ast alle religiösen Biographien, vom 12. bis ins 14. Jahrhundert, haben in einer solchen Relation des Seelenführers zur geistlichen Tochter den Anlaß zu ihrer Entstehung. Denn in solchen höchst subjektiven Erregungszuständen war eine wechselseitige intime Aussprache ein Bedürfnis «. ${ }^{46}$ Dennoch sollten die mit der cura monialium betrauten Priore nur Predigermönche als Beichtväter zulassen, die Fachkompetenz, sittliche Reife und Unvoreingenommenheit gegenüber den Nonnen besäßen. Denn gerade das hinter vergitterten Fenstern zu führende Beichtgespräch bedingte eine heikle Nähe zwischen den Beteiligten und war streng reglementiert, um "persönliche Kontakte oder gar engere Bindungen zwischen den Dominikanerinnen und ihren Seelsorgern zu unterbinden. Geistliche Freundschaften wurden von Seiten der Ordensleitung nicht gerne gesehen, da sie die Gemeinschaft unter den Frauen wie auch die Disziplin empfindlich stören konnten. ${ }^{47}$

Auch die Figur des Beichtigers, obgleich dessen Biographie im Mittelpunkt steht, entzieht sich einer klaren, außerliterarischen Verortung. Man sieht deutlich, »dass hier reale geschehnisse zu grunde liegen. Aber mit grosser geschicklichkeit ist zugleich um sie ein schleier gezogen, der uns diese grundlagen doch nicht in erwünschter schärfe sehen lässt. ${ }^{48}$ Ausgehend von der Ordenszugehörigkeit des Beichtigers und der exponierten Nennung des Ordensgründers Dominikus im

Apokryphen in deutscher Übersetzung und mit Einleitungen, Tübingen u. Leipzig 1904, Kapitel XXIII. Paulusakten, S. 372, Z. 20 f. und S. 373, Z. 32 ff.).

45 Man denke hierbei an Elisabeth von Thüringen und Konrad von Marburg, aber auch Heinrich von Halle und Mechthild von Magdeburg, Heinrich Seuse und Elsbeth Stagel, Heinrich von Nördlingen und Margarete Ebner, Konrad von Füssen und Christine Ebner, Raimund von Capua und Katharina von Siena sowie Petrus Abaelardus und Heloïse; gerade an die beiden zuletzt Genannten fühlt sich Stammler [Anm. 3] mit Blick auf die GR erinnert: »Es ist das gleiche beliebte Thema, wie die Nonne den Beichtvater mit Fragen angeht, nur hier aufgeschwellt zu einem kleinen Roman, zu dem Abälard und Heloise hätten Vorbilder sein können« (Sp. 977). »Daß ein außergewöhnliches Vertrauensverhältnis zu Predigermönchen nicht auf diese besonderen Fälle beschränkt war, zeigen die Briefregister der Generalmagister des späten 14. und 15. Jahrhunderts: Sie enthalten eine große Zahl von Privilegien, die sich Nonnen ausfertigen ließen, um mehrmals im Jahr bei einem Bruder ihrer Wahl die Beichte ablegen zu können« (Ehrenschwendtner [Anm. 43], S. 251 f.).

46 Misch [Anm. 19], S. 92 f.

47 Ehrenschwendtner [Anm. 43], S. 243.

48 Spamer [Anm. 2], S. 378. 
1. Kapitel (Bl. 92 ${ }^{\mathrm{r}-\mathrm{v}}$ ) ist eine dominikanische Provenienz der GR jedoch naheliegend. Die den Dialogtraktat unikal überliefernde Handschrift mit der Signatur Ms. Praed. $159^{49}$ stammt zudem aus dem Besitz des 1233 gegründeten Dominikanerklosters zu Frankfurt/Main. Obwohl ihre schwäbische Schreibsprache auf süd(west)deutsche Herkunft hinweist, nimmt es nicht wunder, dass der Konvent in den Besitz der Handschrift gelangte. Es bilden nämlich jene dort letztlich aufbewahrten Handschriften eine umfängliche Gruppe, die Konventsangehörige in ihrer Studien- und Amtszeit, mitunter weit außerhalb Frankfurts, für den persönlichen Gebrauch ansammelten und die spätestens nach deren Tod der Klosterbibliothek einverleibt wurden. "Bemerkenswert sind insbesondere die engen Beziehungen [...] zum deutschen Südwesten (Elsaß, Schwaben). ${ }^{50}$ Neben der generell für Dominikaner typischen Lebensform (Studium und Seelsorge) werden die Angaben über die Tätigkeit des Beichtvaters der GR mitunter sehr konkret. So erinnert er an eine von ihm selbst gehaltene Predigt über die Ritterschaft Jesu Christi, typischerweise während der Karwoche (2. Kapitel):

>Nun, min vil liebes kind, ich hab nun dir gesait, das ich mich selber wil gesait haben, vnd wil dich weßen mit den waffen vnsers heren vnd wissen in die riterschaft vnd brůderschaft Cristi, als ich in der karwochen prediget: Die durne kronn leg vff wider hofart, das sper wider widernikait, die gaislen wider zorn, die claider Cristi wider gitikait, das crütz mit naglen wider traghait, das grab wider vnluterkait. Also bistu gewafnet in den strit. Aber got gesegen dich vnd sy din widerstands $\left(\mathrm{Bl} .109^{\mathrm{v}}-110^{\mathrm{r}}\right) .^{51}$

49 Ausführliche Beschreibung bei Powitz [Anm. 34], S. 353 ff.: Die 160-blättrige, schwäbische Papierhandschrift stammt aus dem 3. Viertel des 15. Jahrhunderts (Bl. 1-76 sind 1469, Bl. 77-159, darin die GR, etwa gleichzeitig abgeschlossen); auf dem Rückendeckel wird in Einträgen des Klosterbibliothekars Franciscus Jacquin (1707-1776) das Alter der Handschrift im Jahr 1706 auf 237, im Jahr 1735 auf 266 Jahre berechnet. Auf Bl. $2^{\mathrm{v}}-4^{\mathrm{v}}$ trug J. F. Böhmer im 19. Jahrhundert eine Inhaltsangabe ein. Der gebräunte und abgegriffene Zustand von $\mathrm{Bl} .77^{\mathrm{r}}$, dem Textbeginn der GR, deutet auf eine vom vorangehenden Textbestand getrennte, längere Aufbewahrung als Faszikel. Interessant ist der Wechsel der Bastardahände mitten im Text des 1. Kapitels der GR (Bl. $77^{\mathrm{r}}-93^{\mathrm{r}}$ gegenüber Bl. $93^{\mathrm{r}}-153^{\mathrm{r}}$ ). Zur Mitüberlieferung siehe S. $651 \mathrm{ff}$.

50 Powitz [Anm. 34], S. XXIII.

51 Das Motiv von der Ritterschaft Jesu Christi wird eigens in einem gleichnamigen Prosatraktat abgehandelt, der in Ms. Praed. 159 direkt in Anschluss an die GR überliefert ist (Bl. 149 ${ }^{\mathrm{r}}-152^{\mathrm{v}}$ ). Dieser mehrfach überlieferte Traktat bietet speziell Anleitung »zu geistlichen Übungen während der Karwoche« (Monika Kasper-Schlottner: [Art.] Ritterschaft Jesu Christi, in: ${ }^{2}$ VL, Bd. 11, 2004, Sp. 1329), vom Palmsonntag bis Osternacht. Dabei entspricht jedem Tag eine Tugend, für die bzw. eine Todsünde, gegen die es ritterlich zu streiten gilt. Im Verbund mit Gebeten und symbolischen Handlungen (Niederknien usw.) soll man als geistliche Waffe jeden Tag ein Symbol der Passion Christi in Herz und Gedanken tragen: Dornenkrone, Speer, Geißel, Jesu Kleider, Geldbeutel des Judas, Kreuz mit drei Nägeln, Essigschwamm, Grab Christi mit den weißen Leinentüchern. »In Ms. Praed. 159 folgt jeder geistlichen Übung die allegorische Beschreibung der zu bekämpfenden 
Als Standard für diesen Abschnitt des Kirchenjahres ist eine Predigt über die Ritterschaft Christi hinsichtlich der individuellen Autorschaft eines möglichen, historischen Beichtvaters wenig aussagekräftig. Von großem Interesse ist daher der im 6. Kapitel enthaltene >Literaturexkurs`, ein kurzer Katalog von geistlichen Werken, an denen der Beichtvater nach eigener Auskunft arbeitet. Vor ihm liegt ein beachtliches Arbeitspensum, das ihm wenig Zeit für die Seelsorge übriglässt:

Sy sprach: Lieber vater vnd freund gotes, ist vczet besers wen beraitschaft zu dem hailgen sacrament? Der maister sprach: `Ich bin fast vnmüsig: Ich mach ietzunt ain bůch vß ainem volkomen man vnd von ainer starken frauen ${ }^{52}$ vnd ain bůch wider nun sind ${ }^{53}$ vnd ain bůch von siben gaisten von minem ellende. Die tochter sprach: >Haust mich nie gelausen, bist du fast vnmüssig. So sag diner vnmůß ainer (Bl. 135 $)$.

Todsünde; ähnlich wie die bildhaften Darstellungen im `Etymachietraktat` werden sie hier beschrieben als auf verschiedenen Tieren reitend mit Tieremblemen auf Helm und Schild und bestimmte symbolische Gegenstände in der Hand haltend. Möglicherweise gehen diese Textpassagen auf bildliche Darstellungen zurück; zumindest deutet die Aussage Bl. $150^{\mathrm{v}}$ über einen dieser Gegenstände, [...] in der Hand ainen hagen oder ist ain krewal [...] darauf hin. [...] In den anderen Hss. fehlen diese Allegorien, stattdessen heißt es im Vorspann, daß die >R. J. Ch.८ einem Kartäuser in welschen Landen offenbart worden sei« (ebd., Sp. 1330 f.).

52 Bei >De muliere forti könnte es sich um einen Kommentar zu Prv 31,10-31 handeln. Entsprechende Traktate sind für Beda Venerabilis († 735, ১De muliere forti libellus`, in: Jacques Paul Migne [Hg.]: Patrologia cursus completus, series latina, Bd. 91, Sp. 1039-1052) und den Dominikaner Albertus Magnus († 1280, >Liber de muliere forti<, ed. Auguste Borgnet, Bd. 18 [Paris 1893], S. 1-242) überliefert, mit Ms. Praed. 100 (1471/80, siehe Powitz [Anm. 34], S. 233-235) übrigens auch im Frankfurter Dominikanerkloster. Nicht nur über diesen Werktitel, sondern auch über die nur im Prolog der GR erwähnte, allerdings erfolglose Bewerbung um ein Bistum (siehe S. 630) lässt sich eine, wenngleich sehr schwache Verbindung zum Dominikaner Albertus Magnus herstellen; ob dieser dem Beichtiger der GR wirklich Modell stand, ist jedoch mehr als fraglich: Albertus wurde 1260 von Papst Alexander IV. zum Bischof von Regensburg ernannt. Obwohl es die Ordensleitung mit dem Armutsideal als unvereinbar erachtete, hatte der Dominikaner dieses Amt bis 1262 inne. Auf eigene Bitte von Papst Urban IV. davon entbunden und im selben Jahr zum Kreuzzugsprediger ernannt, propagierte er dann aber, gemeinsam mit Berthold von Regensburg, 1263/64 in Deutschland und Böhmen den Kreuzzug (vgl. W. Kübel: [Art.] Albertus Magnus, in: Robert-Henri Bautier [u. a.] [Hgg.]: Lexikon des Mittelalters, Bd. 1, 1980, Sp. 294-299). Der Kirchenvater Sophronius Eusebius Hieronymus (347-420) schließlich verfasste einen Trostbrief unter dem Titel >Ad amicum aegrotum. De viro perfecto< (PL 30, Sp. 75-104).

53 Diese neun fremden Sünden werden im 1. Kapitel (Bl. 92 $)$ kurz erwähnt. Ein entsprechender lat. Traktat `De novem peccatis alienis` (1459, unikal überliefert in Wien, Österreichische Nationalbibliothek, Cod. 4256, Bl. 1a-74a) ist aus der Feder des Georgius Tudel († nach 1465), zeitweise Dekan der Artisten- und theologischen Fakultät sowie Rektor der Universität Wien, zwar bekannt, als Weltgeistlicher stand er aber wohl nicht Modell für den dominikanischen Beichtiger der GR (vgl. Hans-Jochen Schiewer: [Art.] Tudel, Georg, von Giengen, in: ${ }^{2} \mathrm{VL}, \mathrm{Bd} .11$, 2004, Sp. 1566-1568). 
Die Überlieferung geistlicher Werke auf Latein oder in der Volkssprache kennt tatsächlich Texte, die den vier genannten durchaus entsprechen könnten; auf der Grundlage obiger Angaben seien sie unter den folgenden, hypothetischen Titeln aufgelistet: >De viro perfecto<, >De muliere forti<, >De novem peccatis

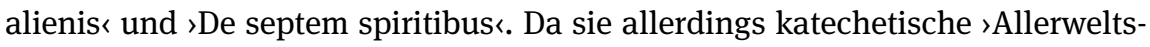
themen dieser Werke betrifft, eher skeptisch: »Ob diese bücher wirklich geschrieben wurden, weiss ich nicht. Mir sind sie nicht bekannt. Doch wäre hier vielleicht der günstigste angriffspunkt für versuche, die persönlichkeit des meisters der >goldenen regel« zu bestimmen. “54 Ein 1434 noch lebender Dominikaner, der genau diese Werke verfasste und darüber hinaus die in der GR geschilderte Biographie vorzuweisen hat, lässt sich nicht ermitteln, immerhin sind die Handschriften zahlreich, die derartig betitelte Textstücke anonym überliefern. Allein der Traktat über die neun fremden Sünden führt zu Johannes Herolt († 1468), Beichtiger (1436) und Generalvikar (1451) der Dominikanerinnen von St. Katharina in Nürnberg und Prior (1438) des dortigen Predigerklosters, als einem besonders sheißen Kandidaten` für die Figur des geistlichen Vaters; allerdings erlebte er nachweislich keine vergleichbaren Krisenmomente und begrenzte sein Wirken auf Nürnberg. Er verfasste aber den lateinischen Traktat >De eruditione Christi fidelium seu de doctrina christiana` (1416), eine Erklärung katechetischer Hauptstücke, darunter der neun fremden Sünden, die mit demselben Bibelzitat (Mt 19,17) beginnt wie die GR. Zudem ist eine Nachschrift deutscher Predigten erhalten, »die er 1436 im [...] Katharinenkloster hielt: ein Zyklus von Adventspredigten [...], eine Weihnachts- und eine Neujahrspredigt. ${ }^{55}$ Warum sollte er daher nicht auch wie der Beichtiger der GR einmal in der Karwoche von der Ritterschaft Christi gepredigt haben?

Der Dominikaner Wenzeslaus von Frankenstein († 1486), mehrfach Prior des Frankfurter Konvents sowie ein unermüdlicher Schreiber und Sammler von Büchern, ${ }^{56}$ teilt mit dem Beichtiger der GR nicht nur die auch in seiner Bio-

54 Spamer [Anm. 2], Sp. 379.

55 Franz Josef Worstbrock: [Art.] Herolt, Johannes (Discipulus), in: ${ }^{2}$ VL, Bd. 3, 1981, Sp. 1123-1127, hier Sp. 1127. Der eingangs zitierte Bibliothekskatalog führt Handschriften auf - Nürnberg, Stadtbibliothek, Cod. Cent. VII, 57 unter Signatur O XXIIII und eine weitere unter M XII -, die Predigten Herolts enthalten (siehe MBK [Anm. 4] S. 635, Z. 21 f. sowie S. 626, Z. 20 ff.).

56 Siehe Powitz [Anm. 34], S. XX f. In etlichen, beinahe ausschließlich lateinischen Handschriften des Frankfurter Dominikanerklosters tritt magister Wenzeslaus als Käufer, sehr viel häufiger jedoch als Schreiber, Rubrikator, Kommentator oder Initiator für deren Entstehen in Erscheinung: Ms. Praed. 29, 95, 118, 121 (die darin enthaltenen, deutschen Textstücke sind in Faszikeln überliefert, die nicht mit Wenzeslaus in Verbindung stehen), 122, 124, 128, 133, 136, 138, 
graphie verbürgte Beicht-, Studien- und Lehrtätigkeit ${ }^{57}$, sondern kennt, da auch er in Konflikt mit dem Orden geriet, vergleichbare Krisenmomente: 1465 unterstützte er den Klostervikar Heinrich Schretz bei der langwierigen und hart umkämpften Reformierung des Dominikanerinnenklosters St. Agnes in Straßburg, wo er auch als Beichtiger der Ordensschwestern von St. Nikolaus in undis eingesetzt war. Der Ordensgeneral Marcial Auribelli (1452-1463 und 1465-1473), zunächst nicht von der Notwendigkeit der Reform überzeugt, bestätigte diese zwar zunächst, bestrafte jedoch Schretz und Wenzeslaus für ihr Unruhe stiftendes Vorgehen bei der Reformierung des Klosters. Wenzeslaus verlor daraufhin sein Beichtigeramt. ${ }^{58}$ Eine Autorschaft der in der GR genannten Werke ist für Wenzeslaus nicht verbürgt. Es ist jedoch denkbar, dass er für die Entstehung der GR eine impulsgebende Rolle spielte, bewegte er sich doch auch im Süden des deutschsprachigen Raums und reformierte das Frankfurter Dominikanerkloster. Somit könnte er das fehlende Bindeglied zwischen Entstehungsraum (vermutlich Schwaben) und Aufbewahrungsort (Frankfurt) der GR in Ms. Praed. 159 darstellen, indem er etwa die Abschrift einer im Süd(west)en entstandenen Vorlage des Dialogtraktats oder gar die Abfassung der GR selbst in Auftrag gab und, in beiden Fällen, das Werk später nach Frankfurt brachte. Seine eigenhändig datierten und lokalisierten Handschrifteneinträge bezeugen für die 60er- und 70er-Jahre des 15. Jahrhunderts, dem Entstehungszeitraum von Ms. Praed. 159, eine Konzentration auf die betreffende Region - Colmar (1461), Straßburg (1465/ 66 und 1471/72) und vor allem das schwäbische Ulm (1466) - was sich gerade in der Schreibsprache der Handschrift widerspiegeln könnte.

161 und 176 (siehe jeweils Powitz, vor allem S. 314 zur Gruppe der Wenzeslaus-Handschriften). Die von seiner Hand geschriebenen Texte und Einträge sind zumeist datiert, lokalisiert sowie mit Namenssigel versehen. Überschneidungen mit Textstücken der GR bietet einzig Ms. Praed. 138, das auf Bl. $139^{\mathrm{v}}-140^{\mathrm{r}}$ die >Consilia Anselmi circa hominem moriturumı enthält (ebd., S. 318). Bl. $145^{\mathrm{v}}$ und $\mathrm{Bl} .146^{\mathrm{r}}$ der GR führen dementsprechend die sieben Fragen des Anselm von Canterbury an einen Sterbenden auf.

57 Wenzeslaus immatrikulierte sich 1447/48 an der Universität Köln, promovierte 1450 zum Dr. theol. und wurde dort 1462 Regens; vgl. Powitz [Anm. 34], S. 314 und Benedict Maria Reichert (Hg.): Acta Capitulorum Generalium Ordinis Praedicatorum, Bd. 3: Ab anno 1380 usque ad annum 1498, Rom 1900 (Monumenta Ordinis Fratrum Praedicatorum historica 8), S. 284, Z. 29 f. 58 Siehe Benedikt Maria Reichert (Hg.): Buch der Reformacio Predigerordens, Buch IV-V, Leipzig 1908 (Quellen und Forschungen zur Geschichte des Dominikanerordens in Deutschland 3), Buch V, Kapitel 62, 64 f. und 71 sowie Annette Barthelmé: La réforme dominicaine au XV siècle en Alsace et dans l'ensemble de la province de Teutonie, Straßburg 1931, S. 82 ff.). 
Seit Williams-Krapps ${ }^{59}$ Beitrag über Heinrich Seuses >Vita`, lange Zeit als »erste[s] Beispiel einer vom Helden selbst verfassten, bzw. autorisierten und herausgegebenen Autobiographie in deutscher Sprache ${ }^{60}$ missverstanden, ist höchste Vorsicht bei der biographischen Lesart vermeintlich (auto-)biographischer Texte geboten, so auch im Fall der GR. Müller schlägt daher vor, unter Einbezug von außerliterarischen Quellen, literarischen Traditionen und Erzählmustern, »die (literarisch-poetische) Transformation der erlebten Realität durch die Darstellung sowie [...] die zugrundeliegende Absicht des Autors und - unmittelbar damit zusammenhängend - die beabsichtigte Wirkung (Motivation) « ${ }^{61}$ solcher Texte in den Blick zu nehmen. Ausgehend von der augenfälligen Diskrepanz zwischen Seuses dominikanischer Lebensform und der Stilisierung des Dieners zum QuasiEremiten zeigte Williams-Krapp, dass Seuses >Vita ‘ mit Motiven und Erzählstoffen der >Vitaspatrum $`$ nachgerade durchsetzt ist. Seuse intendiere mit seinem Erzählwerk keine Verherrlichung der eigenen Person, sondern eine "pädagogisch behutsame Aufbereitung zentraler Aspekte der Altväterspiritualität für Kreise, die nach neuen Formen vertiefter religiöser Lebensführung drängten. Dem `Seelenführer`Seuse erschien es daher erstrebenswert, den Schwestern nicht die historischen Texte direkt, sondern quasi kommentiert in Form aktueller Lebensbeispiele $\mathrm{zu}$ vermitteln “2 $^{62}$. Eine vergleichbare Diskrepanz zwischen Lebensform und Darstellung liegt in der GR nicht vor: Seinem sapostolischen Auftrag ‘ prinzipiell treu, nimmt der Beichtiger im Wechsel von Seelsorge und Studium einen typisch dominikanischen Lebensweg, entzieht sich zeitweise jedoch durchaus seinem seelsorgerisch $\mathrm{zu}$ betreuenden Umfeld und erlebt, in Übereinstimmung mit innerer Entwicklung sowie in Reaktion auf Impulse von außen, Schwankungen, die ihn in seinem Leben oftmals unvorhergesehene Abzweigungen gehen lassen. Auch wenn Diener und Beichtiger unterschiedlich zur Darstellung kommen mögen, ${ }^{63}$ so könnte Seuses >Vita - Vorbild für erbauliche Reflexionsauto-

59 Vgl. Werner Williams-Krapp: Nucleus totius perfectionis. Die Altväterspiritualität in der >Vita` Heinrich Seuses, in: Johannes Janota [u. a.] (Hgg.): Festschrift Walter Haug u. Burghart Wachinger, Bd. 1, Tübingen 1992, S. 407-421.

60 Karl Bihlmeyer (Hg.): Heinrich Seuse. Deutsche Schriften, Stuttgart 1907 (ND 1961), S. 135*.

61 Müller [Anm. 16], S. 430.

62 Williams-Krapp [Anm. 59], S. 419.

63 Heinrich Seuses (1295/97-1366) >Vita bietet in Kapitel 21-32 eine mit der GR vergleichbare, novellistische Schilderung äußeren Leids, dem sich der Diener, von der Stufe des in- zum proficiens aufgestiegen, im Anschluss an die Fußtuchszene geduldig stellt; mit diesem von außen kommenden Leid ist der hiesige Beichtiger, der den Umweg des Dieners über die Radikalaskese nicht nimmt, zwar von Anfang an konfrontiert, erkennt dessen heilsmäßigen Nutzen jedoch erst spät (6. Kapitel), nach Ablauf vieler kleiner Etappen geistlicher Entwicklung bzw. an der im `Lehrplan` der GR vorgesehenen Stelle geistlicher Progression. 
biographie $^{64}$ - der GR durchaus Modell für die Literarisierung eines geistlichen Lebenslaufs gestanden haben, die den typischen Werdegang eines Dominikaners aus katechetisch-didaktischen Gründen hier zur `Pseudo-Biographie` verfremdet. Oder mit Stammler kurz gesagt: »An Nacherzählung eines wirklichen Ereignisses glaube ich nicht, sondern halte das Ganze für eine Erfindung in Dominikanerkreisen, aus denen auch die Handschriften herkommen. ${ }^{65}$ Sie zeichnet sich dabei durch Überlagerung und Vermischung von biographischen Elementen mit Motiven und Themen der literarischen bzw. legendarischen Tradition aus, im Fall der >Vita $<$ mit den >Vitaspatrum $<$, im Fall der GR mit der Legende der Thekla von Ikonium. Doch welche Vermittlungsstrategie verfolgt die Lebensschilderung eines >Prototyp-Dominikaners ' gegenüber ungebildeten Adressaten, die nicht dieser Lebensform angehörten und deren Identifikationsfigur vielmehr die nur mit der Nebenrolle besetzte Beichttochter darstellte? Sicherlich standen die GR sowie alle Texte in Ms. Praed. 159 erstrangig im Dienst der Seelsorge von Dominikanern an den ihnen anvertrauten Dominikanerinnen, die sich auch durch Bereitstellung geistlichen Schrifttums in der Volkssprache ausdrückte. Für diese, aber auch für außermonastische simplices bietet die GR biographisch eingebettete Belehrungen für die tägliche Frömmigkeitspraxis und Vorbilder für die gottgefällige Bewältigung von Lebenskrisen und geistlichen Skrupeln, und zwar im wahrsten Sinne des Wortes vorgelebt, in Übereinstimmung von vita und verba und scheinbar, da pseudobiographisch, mit der Autorität lebenspraktischer Erfahrung: Der Beichtvater habe die bichttochter nie nit gelert, er ervolgte es vor an im selber (Bl. $\left.126^{\mathrm{V}}\right)$. Diese Art von Katechese erweist sich als besonders lebensanschaulich, wird sie doch am Werdegang eines lese- und lebemeisters vorgeführt, dem etwa Dominikanerinnen, für die Ms. Praed. 159 vermutlich zusammengestellt wurde, dergestalt im klösterlichen Alltag von Predigt und Beichte tatsächlich begegnen konnten. Hinzu kommt sicherlich auch der besondere Reiz, den die Fallhöhe nicht eines den simplices ebenbürtigen, einfachen Gläubigen, sondern eines geistlichen Lehrers und Beichtigers ausmacht, der mit inneren und äußeren Widrigkeiten des Lebens umgehen muss und dabei, freilich nur rein theoretisch, scheitern könnte. Dass die GR dabei auch auf den monastischen Personenumkreis ihres Verfassers vorbildlich und nachahmenswert wirken konnte, ist damit nicht ausgeschlossen.

64 Müller [Anm. 16], S. $431 \mathrm{f}$.

65 Stammler [Anm. 3], Sp. 977. 


\section{Entstehung, Kurzfassung und Mitüberlieferung der iguldin regels}

Die Überlieferung der >guldin regel in Dominikanerkreisen lässt auf eine Entstehung in gleichem Umfeld schließen. Dies gilt auch für die Kurzfassung, die, im Gegensatz zur in Ms. Praed. 159 unikal überlieferten GR als umfangreicher Ausarbeitung des Motivs der Goldenen Regel, zumeist nur die sieben, auch im Prolog der GR aufgelisteten Gebote enthält (siehe S. 626 f.). Allein aufgrund des handschriftlichen Befunds lässt sich über die Vor-, Gleich- oder Nachzeitigkeit im Verhältnis zwischen GR und Kurzfassung nichts aussagen. Er gibt jedoch Anlass zur Vermutung, dass es letztere bereits vor 1440/50 gegeben haben muss - auf diesen Zeitraum ist die älteste Handschrift datiert -, während die GR aus heutiger Perspektive erst um das Jahr 1469 herum in handschriftlicher Überlieferung zu Tage tritt. Bislang konnten drei Handschriften ausgemacht werden, welche diese wohl zeitlich vor der GR entstandene Kurzfassung überliefern:

1. Nürnberg, Stadtbibliothek, Cod. Cent. VI, 43m, Bl. $292^{\mathrm{r}-\mathrm{v}}$

ca. 1440-1450, im Nürnberger Katharinenkloster geschrieben ${ }^{66}$, geistliche Sammelhandschrift (u. a. Johannes Nider, Johannes Tauler), nürnbergisch (Bl. 1-18 $8^{\mathrm{v}}, \mathrm{Bl} .46^{\mathrm{r}}-60^{\mathrm{v}}$ ), Bl. $198^{\mathrm{r}}-308^{\mathrm{v}}$ alemannisch gefärbt; siehe Schneider [Anm. 5], S. 112-117.

2. Augsburg, Universitätsbibliothek, Cod. III.1.4 ${ }^{\circ} 8, \mathbf{B l}^{2} 238^{\mathrm{r}}-239^{\mathrm{r}}$

1464, Zisterzienserinnenkloster Kirchheim im Ries, von Johannes K. von Bopfingen geschrieben, geistliche Sammelhandschrift (u. a. Thomas Peuntner, Johannes Nider, Konrad Kügelin) mit erbaulichen Traktaten und Kurztexten, die Kurzfassung der GR ist zwischen Exzerpten aus Mechthilds von Magdeburg >Das fließende Licht der Gottheit (Bl. $236^{\mathrm{v}}-238^{\mathrm{r}}$ und $\mathrm{Bl}$. 239 ${ }^{\mathrm{r}}-242^{\mathrm{r}}$ ) eingetragen; stark bairisch gefärbt; Erstbesitz: Gräfin Agnes von Werdenberg († nach 1471), Mutter der Reformäbtissin Magdalena von Oettingen (1424-1502); siehe Schneider [Anm. 68], S. 264-271.

3. München, Staatsbibliothek, Cgm 470, Bl. $69^{\mathrm{v}}$

2. Hälfte 15. Jahrhundert, Schwaben, Herkunft unbekannt, 70 Bll., Text der letzten Ternionenlage von 7. Hand (Bl. 65-69) ostschwäbisch, geistliche Sammelhandschrift (`Tochter von Sion`, Allegorien u. a.): ‘Geistlicher Maibaum` (Bl. $1^{\mathrm{r}}-15^{\mathrm{r}}$ ), Geistliche Regeln (bei Schneider: `Lebensregeln für eine Beichttochter`, Bl. $35^{\mathrm{r}-\mathrm{v}}$ ), die Bitten des Paternosters nach den sieben Tagzeiten $\left(\mathrm{Bl} .70^{\mathrm{r}-\mathrm{v}}\right.$ ); siehe Karin Schneider: Die deutschen

66 Der eingangs erwähnte Bibliothekskatalog führt auch diese Handschrift, unter der Signatur E XXXV, auf: Item ein news puch; das helt in im von worer, | sicher andacht und von sant Anna, | IIII frog von der mynnenten sel, ein schöne | ler von der mynnenten sel, ein predig von der myn- | nenten sel ůber die IIII wach, die sie getan hat, | und scho̊ner predig IIII von mynnen und das | püchlein, das da heist Des ewigen lebens in | dem namen Jhesu Cristi, und die gulden regel | und scho̊n sprüch der lerer und XXV czaichen | eins waren, vermüftigen gruncz. | Das puch haben die swester geschriben (MBK [Anm. 4], S. 607, Z. 37 - S. 608, Z. 4). 
Handschriften der Bayerischen Staatsbibliothek München. Cgm 351-500, Wiesbaden 1973 (Codices codicum manu scriptorum Bibliothecae Monacensis V,3), S. 387-390.

Bemerkenswert an dieser überschaubaren Überlieferung ist erstens, dass der älteste Überlieferungszeuge, die Nürnberger Handschrift aus dem Dominikanerinnenkloster St. Katharina, entgegen den übrigen darin aufgezeichneten Texten gerade an der Stelle alemannisch gefärbt ist, an der die Kurzfassung der 'guldin regel eingetragen ist. Zwar sind Ms. Praed. 159 und Cgm 470 (ost-)schwäbisch, doch verweist jene Färbung durch eine dem Schwäbischen benachbarte Sprachlandschaft gleichermaßen auf den Süden des deutschsprachigen Raumes als Entstehungsort des Motivs der Goldenen Regel in Kurz- u n d Langfassung. Diese schreibsprachliche Nähe zwischen Ms. Praed. 159 und Cent. VI, 43m, beide aus dominikanischem Umfeld, macht eine Entstehung auf schwäbisch-alemannischem Boden daher sehr wahrscheinlich. Gelangte die Kurzfassung demnach schon früher, vor 1440-50 nach Nürnberg, wo sie, so scheint es, von einer alemannischen Vorlage abgeschrieben wurde, so ist es denkbar, dass sich die Ausarbeitung zum Dialogtraktat im alemannischen oder schwäbischen Raum vollzog und über die anskizzierten Ordens- und persönlichen Beziehungen schließlich Frankfurt erreichte; die Augsburger Handschrift deutet freilich auf eine Ausbreitung des Motivs im bairischen und damit dem Schwäbischen ebenfalls benachbarten Raum hin. Zweitens sind die Rezipienten der Kurzfassung gerade in Frauenkonventen, nicht nur des Dominikanerordens, zu finden: St. Katharina (Nürnberg) und Kirchheim im Ries; dies wird auch für die GR anzunehmen sein. Die Kurzfassung der sguldin regel sei auf Grundlage der drei genannten Handschriften synoptisch dargestellt: ${ }^{67}$

Cent. VI, 43m, Bl. 292 ${ }^{\text {r-v }}$ :

Dises ist die guldin regel vnd hat acht capitel. Das erst capitel: Du solt dick vnd oft beichten luterlichen. Das ander: ... vnd dich huten vor allen sunden steticklichen. Das drit: Du solt dick vnd vil gen zu dem heiligen vnd lebendigen sacrament andechticlichen. Das virde: ... vnd dich darzu alzit bereiten minsamlichen. Das fvnft: Dv solt alle tvgent vben
Cod. III.1.4 ${ }^{\circ}$ 8, Bl. 238 ${ }^{\mathrm{r}}-239^{\mathrm{r}}$ :

Dv solt dick vnd vnd vil lauter-

lich peichten. Du solt dich hütten vor newem peichten vnd vor newen sünden. Du solt als vil tugend wben, als du vntugend gewbt hast. Wann wa ain sunde stönd, da sol nw ain tugend stan. Du solt dick vnd vil zú dem hailigen sacrament gan mit urlob. Du solt dich all tag beraitten zü dem sacrament, als solltest du all tag
Cgm 470, Bl. 69v:

Die gulden regel: Du solt fül vnd dick peichten. Du solt dich huetten vor newen beichtten vnd vor newen sünden. Du solt als fül tugent üben, als füll du vndugent geübt hast. Vnd wa ain sund stünd, da sol nun ain tugent stan. Du solt fül vnd dick zü dem hailligen sacrament gan mit vrlab. Du solt dich altag beraiten zü gan. Du solt gan in die batstüben des

67 Zum Motiv der Badstube siehe S. 631, Anm. 23; zur Bedeutung von hag und hurst siehe S. 631, Anm. 24 und 25. 
getrvlichen. Das sechst: ... vnd teglichen in die batstvben gan geistlichen. Das ist, das liden vnd das leben Cristi betrachten hicziklichen. Das sibent: Du solt laufen durch den hage gedulticlichen. Das ist nicht verzagen noch verzwifeln in liden vnd in bekorunge. Das achte: ... vnd gern predige horen erwirdiclichen.

Amen. zügan. Du solt gan jn die padstuben des ölpergs vnd heraus schwiczen dein aigen willen, als Cristus tett. Durch den hag, durch den zaun, durch die hurst so chompst du in denn gartten durch alles stechendes leiden etc. elpergs vnd her auß schwitzen dein aigen willen, als Cristus det. Durch den hag, durch den zün, durch die hurst so kumst du in den gartten durch alles stechenden leiden.

Die Kurzfassung ist, wie ersichtlich, in zwei Redaktionen überliefert: Die Nürnberger Handschrift steht allein gegen den Augsburger und Münchner Überlieferungszeugen: Cent. VI, 43m nennt insgesamt acht Regeln in der im Prinzip offenen und problemlos erweiterbaren Auflistung von Geboten und verändert ein wenig deren Reihenfolge: Tugendübungen an fünfter, nicht dritter Stelle. Aufgrund ihres geringen Umfangs ist die Kurzfassung freilich nebst einer ganzen Reihe anderer geistlicher Texte überliefert; in Cgm 470 dient die sguldin regel sogar als nachträgliche Einleitung für eine mehrere handschriftliche Blätter umfassende Belehrung einer geistlichen Tochter ${ }^{68}$, die jedoch nicht mit der GR identisch ist.

Die textliche Zusammenstellung in Ms. Praed. 159 unterscheidet sich nicht wesentlich von derjenigen in Handschriften mit Kurzfassung und ist einsichtig: Im Anschluss an die GR finden sich Textstücke wie >Die Ritterschaft Jesu Christi (Bl. $149^{\mathrm{r}}-152^{\mathrm{v}}$, siehe S. 643, Anm. 51) und >Der Seele Baum` (Bl. 153 ${ }^{\mathrm{r}}$ ); bereits das 2. Kapitel der GR bedient sich des Motivs der militia Christi sowie der Auslegung dreier Paradiesbäume nach Hugo von St. Viktor zur Gliederung der Zehn Gebote (Bl. $96^{\mathrm{r}}-98^{\mathrm{v}}$ ), angelehnt an >De fructibus carnis et spiritus` (PL 176 [Anm. 52], Sp. 997-1006). Alle diese Texte sind zudem von einer ab Bl. 93 ${ }^{\mathrm{r}}$ (mitten im 1. Kapitel) einsetzenden, dritten Hand geschrieben worden, was ihre inhaltliche und strukturelle Zusammengehörigkeit unterstreicht. Der Eintrag eines von der vierten Hand aufgezeichneten Glaubensbekenntnisses $\left(\mathrm{Bl} .153^{\mathrm{v}}-155^{\mathrm{r}}\right)$, ebenfalls in Ms. Praed. 170 überliefert, ist unproblematisch; Bl. $155^{\mathrm{v}}-159^{\mathrm{v}}$ sind unbeschrieben. Die Zusammenführung der GR mit Meister Eckharts `Reden der Unterscheidung ‘ (Bl. $5^{\mathrm{r}}-76^{\mathrm{r}}$ ) erfolgte mit etwas zeitlichem Abstand: Bl. $77^{\mathrm{r}}$, das >Deckblatt der GR,

68 Siehe Karin Schneider: Deutsche mittelalterliche Handschriften der Universitätsbibliothek Augsburg. Die Signaturengruppen Cod. I.3 und Cod. III.1, Wiesbaden 1988 (Die Handschriften der Universitätsbibliothek Augsburg II.1), S. 389. 
ist gebräunt und vergriffen, was auf eine länger andauernde und von Eckharts Traktat getrennte Aufbewahrung schließen lässt. Zudem wurden die >Reden einer anderen Hand aufgezeichnet als die GR; Bl. $76^{\mathrm{V}}$ zwischen den beiden Texten ist leer geblieben. Die Zusammenführung liegt wohl schlichtweg darin begründet, dass hier zwei Lehrgespräche dominikanischer Provenienz zusammengestellt werden sollten. ${ }^{69}$ Dies hat jedoch auch dazu geführt, dass man die GR »[z]u Unrecht [...] in die mystische Literatur gerechnet ${ }^{70}$ hat. Denn Spamer attestierte der GR »trotz aller verschiedenheiten viel verwantes « ${ }^{71}$ mit der 〉Schwester Katrei , einem anonymen, mystischen Dialogtraktat, der um 1320, nach Beginn der Straßburger Beginenverfolgung (1317-1319), entstand. Trotz struktureller und inhaltlicher Gemeinsamkeiten - Dialog zwischen Beichtvater und -tochter, Episodenhaftigkeit, novellistische Rahmung, Transparenz der inneren Wandlung und Entwicklung der Beteiligten ${ }^{72}$ - wird der Beichtvater der GR von seiner geistlichen Tochter zu keinem Zeitpunkt derartig übertroffen, dass sie letztlich dazu überginge, den Geistlichen ihrerseits zu belehren. In ১Schwester Katrei` hingegen legt sie selbst modellhaft den vom Geistlichen aufgezeigten Weg zurück, »ähnlich den

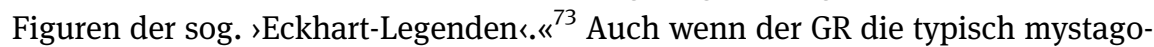
gischen Themen dieser »häufig dialogisierte[n] Exempelgeschichten ${ }^{74}$ gänzlich fehlen, könnten die Kompilatoren von Ms. Praed. 159 dennoch eine gewisse Verwandtschaft mit Eckhart-Legenden nach dem Muster von >Meister Eckharts Tochter` gesehen haben. Sie hätten demnach beide Texte zusammengeführt, begünstigt durch den Umstand, dass »Erzählform, konkrete äußere Umstände

69 In Ms. Praed. 159 sind Meister Eckharts >Reden der Unterscheidung folgendermaßen überschrieben: Das sind die rede, die der vicarius von düringen, der prior von erdfort vnd brůder eckhart prediger ordens mitt sôllichen kindern het, die in diser rede fragten vil dings, do sy sassen in collacionibus mit ainander $\left(\mathrm{Bl} .5^{\mathrm{r}}\right)$. Dieses Werk, während Eckharts Erfurter Priorats entstanden, enthält Lehrgespräche zwischen dem Prior und jüngeren Brüdern über zentrale Fragen des religiösen Lebens von Ordensleuten. »In drei Kapitelgruppen werden das Gebet, die Suche und Verwirklichung der Gelassenheit, Selbstprüfung, das Wachsen auf Gott hin (Kap. 1-8), die Sünde (Kap. 9-16) und Fragen der konkreten Lebensführung in der Nachfolge Christi (Kap. 7-23) besprochen« (Bruno Jahn: [Art.] Meister Eckhart, in: Deutsches LiteraturLexikon [Anm. 1], Sp. 7-23, hier Sp. 11).

70 Stammler [Anm. 3], Sp. 977.

71 Spamer [Anm. 2], Sp. 378.

72 Siehe Franz-Josef Schweitzer: Der Freiheitsbegriff der deutschen Mystik. Seine Beziehung zur Ketzerei der `Brüder und Schwestern vom Freien Geist`, mit besonderer Rücksicht auf den pseudoeckartischen Traktat `Schwester Katrei` (Edition), Frankfurt/Main u. Bern 1981 (Europäische Hochschulschriften. Reihe I: Deutsche Sprache und Literatur 378), S. 168.

73 Ebd.: [Art.] Schwester Katrei, in: ${ }^{2}$ VL, Bd. 8, 1992, Sp. 947-950, hier Sp. 949.

74 Kurt Ruh: [Art.] Eckhart-Legenden, in: ${ }^{2}$ VL, Bd. 2, 1980, Sp. 350-353, hier Sp. 350. 
(Predigerkloster) und bestimmte Figuren wie Pförtner und >Meister Eckhart ‘....] eine reale Handlung, die Charakteristikum der Legende ist ${ }^{75}$, vorgeben, wie es ja auch die GR mit ihren vermeintlich biographischen Elementen tut. Vor allem die zunächst rätselhafte Erklärung der Beichttochter zu Beginn des 3. Kapitels, in Rekurs auf die im 2. Kapitel behandelten, geistlichen Waffen (siehe S. 636 und S. 643), erinnern an die >Legende $\iota:$

\footnotetext{
Ein tohter kom ze einem predierklôster und vordert meister Eckeharten. Der portenêre sprach svon weme sol ich im sagen? Si sprach sich enweiz.« Er sprach swâ von wizzent ir sîn niht?»Si sprach >dâ enbin ich niht ein maget noch ein wip noch ein man noch ein frowe noch ein witwe noch ein juncfrowe noch ein herre noch ein dierne noch ein kneht. ${ }^{76}$
}

Im vorliegenden Fall ist es somit weniger Eckhart als Verfasser der `Reden , der als Bindeglied zwischen beiden Texten fungiert, sondern vielmehr Eckhart als literarisierte Figur eines gelehrten und lehrenden Dominikaners in einer >Legende`, die in vergleichbarer Weise auf den vermeintlichen Realitätsgehalt der Darstellung Wert legt. Meister Eckharts Lebensweg deckt sich freilich kaum mit dem in der GR dargestellten Werdegang des Prototyp-Dominikaners - eine Gleichsetzung beider Ordensbrüder ist daher unsinnig -, doch kommt nach Heinrich Seuse, Johannes Herolt und Wenzeslaus von Frankenstein mit ihm nun ein vierter Dominikaner ins Spiel, der wie der Beichtiger der >guldin regel im Lauf seines Lebens auch äußeren, mitunter ordenspolitischen Widrigkeiten ausgesetzt war. Ihr aller Leben ist gewiss nicht unmittelbare >Vorlage ‘ für die GR, gutmöglich aber Quelle der Inspiration für einen Dialogtraktat, der geistliche Lehre unmittelbar mit dominikanischer Lebensform verknüpft und diese systematisch in sieben Goldene Regeln kleidet.

75 Franz-Josef Schweitzer [Anm. 72], S. 167.

76 Ed. Franz Pfeiffer: Deutsche Mystiker des vierzehnten Jahrhunderts, Bd. II: Meister Eckhart, Leipzig 1857, S. 625,4-9 (Spruch 69). 